\title{
Cloud Control System Architectures, Technologies and Applications on Intelligent and Connected Vehicles: a Review
}

\author{
Wenbo Chu' ${ }^{1}$, Qiqige Wuniri' ${ }^{1}$ Xiaoping Du,2, Qiuchi Xiong ${ }^{1}$, Tai Huang ${ }^{1}$ and Keqiang Li, ${ }^{1,3^{*}}$
}

\begin{abstract}
The electrification of vehicle helps to improve its operation efficiency and safety. Due to fast development of network, sensors, as well as computing technology, it becomes realizable to have vehicles driving autonomously. To achieve autonomous driving, several steps, including environment perception, path-planning, and dynamic control, need to be done. However, vehicles equipped with on-board sensors still have limitations in acquiring necessary environmental data for optimal driving decisions. Intelligent and connected vehicles (ICV) cloud control system (CCS) has been introduced as a new concept as it is a potentially synthetic solution for high level automated driving to improve safety and optimize traffic flow in intelligent transportation. This paper systematically investigated the concept of cloud control system from cloud related applications on ICVs, and cloud control system architecture design, as well as its core technologies development. Based on the analysis, the challenges and suggestions on cloud control system development have been addressed.
\end{abstract}

Keywords: Intelligent and connected vehicles, Cloud control system, Cloud control base platform, Cloud controlled and automated driving

\section{Introduction}

In recent years, as the rapid development of intelligent and connected vehicle (ICV) technology [1], the demand for automated driving application in logistics [2], sanitation [3], port terminals [4], mining [5], retail [6], ride sharing [7], public transportation [8] and other industries has surged and attracted wide attention from both industry and academia. According to the latest Gartner Hype Cycle for Connected and Smart Mobility, most of the technologies related to automated driving and new mobility are in the trough of disillusionment, which means the commercialization of those technologies is underway [9].

\footnotetext{
${ }^{*}$ Correspondence: likq@tsinghua.edu.cn

${ }^{3}$ State Key Laboratory of Automotive Safety \& Energy, School of Vehicle and Mobility, Tsinghua University, Beijing 100084, China

Full list of author information is available at the end of the article
}

The issues of ICV technology development include three main phases. (1) The insufficient reliability of intelligent vehicle perception system causes blind spot. Limited computing resource of intelligent vehicle makes it difficult to adapt to different and complicated road traffic environments. Improving the reliability and computing capability by using more sensors on the vehicle side will greatly increase the cost. Thereby, the road side infrastructures are required and the vehicle-road coordination technology are developed [10]. (2) Although the vehicleroad coordination technology can enhance the vehicle perception capability and improve the safety and driving predictability to a certain extent, it also faces new bottlenecks. Information interaction among different kinds of road-side equipment is still lack of unified standard. It results in a high cost of multi-source perceptive information sharing collected from heterogeneous sensors [11]. Furthermore, the insufficient centralized decisionmaking and control capability of the multi-vehicles also 
restricts the optimization of road traffic efficiency [12]. (3) With the development of cloud computing, big data, internet of things, and wireless communication technologies, a new concept of "ICV cloud control system (CCS)" has been proposed to resolve previous problems [13]. In our opinion, it is a typical application of cyber-physical system (CPS) theory in the field of intelligent and connected vehicles. In this system, vehicle is no longer a type of transportation, but a platform for human-vehicle-road-cloud information transmission and sharing. ICV cloud control system can enlarge the perception range of intelligent vehicles, increase the driving safety of connected vehicles and enhance the existing vehicle-road coordination ability by providing centralized decision-making and control capability via utilizing edge computing, vehicle to everything (V2X) communication and artificial intelligent (AI) technologies [13]. It now becomes the latest trend of ICV technology and influences more and more national automated driving development strategies [14-19].

This paper represents a systematic review on the existing literature of cloud systems, approaches and technologies related to intelligent and connected vehicle control. The structure of the paper is as follows. Section 2 reviews the typical ICV applications that used cloud technology and expounds the concept of cloud control system. Section 3 investigates the CPS (cyber physical system) architecture design and provides the methods that can be utilized to design ICV CCS. Section 4 categorizes the common key technologies of ICV CCS and systematically describes the current state of development and key issues in each technology area. Section 5 concludes the challenges of ICV CCS and gives the development trend of its key technologies.

\section{Cloud Related ICV Applications and Cloud Control Concept}

\subsection{Cloud Related ICV Applications}

With the development of cloud computing, big data, internet of things, and wireless communication, traditional automotive industry is experiencing a new revolution. Especially, the development of ICV based on traditional automotive and transportation industry will introduce much more jobs and promote the economics and society with great potential [20]. Vehicle is no longer a type of transportation, but a platform for human-vehicle-road-cloud information transmission and sharing. Equipped with various types of sensors, vehicles are able to have complex environment perception capability with the implementation of cooperative planning and control. The most critical step in vehicle automation is to realize safe, comfortable driving with high efficiency and low energy consumption. Recently, the researchers have identified the insufficiency of intelligent vehicle and the limitation of Vehicle-to-Infrastructure (V2I) cooperation. To continuously increasing the driving safety, comfort and energy-saving to realize fully automated driving, many countries have been launching policies, carrying out kinds ICV projects, and constructing cloud related ICV systems.

In early 2020, eleven central level Chinese governmental departments jointly issued the Strategy for Innovation and Development of Intelligent Vehicles [21], indicating that China has already set ICV as the core development plan for the country. The U.S Department of Transportation (USDOT) published a series of ITS JPO Strategic Plan [22] and included connected and automated transportation to its emerging and enabling technologies. The European Commission organized and set up cooperative Intelligent Transport Systems (C-ITS) Deployment Platform, which developed a shared vision on the interoperable deployment of the C-ITS towards cooperative, connected and automated mobility (CCAM) in the European Union [23].

As regarding to ICV projects and applications, many of them tend to adopt 5G, cloud computing technologies. Chang et al. [24] mentioned a predictive backward shockwave analysis approach (PSA) that includes both macro and micro PSA models to realize real-time active safe driving under uncertain and high-risk road conditions. The work specifies a three-layer cloud computing mechanism, which are vehicular cloud computing (VCC), Multi-access Edge Computing (MEC), and global cloud computing (GCC). The method uses the analysis and prediction of vehicle driving condition data to identify backward shockwave of traffic flow with high risk. Then, it reduces the risk by using the three-stage cloud computing mechanism to send warning signals to high-risk area. Such method can also be applied with human-driving vehicles. But in this application, the cloud platform is mainly applied for traffic flow shockwave prediction.

Based on the Cloud-Assisted Real-time Methods for Autonomy (CARMA) project, Montanaro and Fallah introduced a cloud platform under mixed traffic scenario for passenger car platoon control and management [25]. The architecture is composed by three layers: Trip-Planner, Road Section Manager (RSM), and Coordination Control. The top layer, Trip-Planner computes the global optimal route and speed profile for the minimization of the energy consumption on each section of the highway system and for all CARMA vehicles operating in any vehicle mode (Adaptive Cruise Control (ACC)-mode, Free Agent (FA)-mode, and Platoon Leader/Platoon Follower (PL/PF)-mode). For vehicles operating in platoons, the Trip-Planner also provides a suggested inter-vehicular distance. This layer requires altitude profile for each 
highway segment, traffic, weather, and road conditions, etc. The RSM is responsible for refining vehicle local speed profiles and inter-vehicular distance (for platoons already formed) to adapt them to current road section state. The layer can also control the speed of FA vehicle and corresponding platoon for merging maneuver. It also needs to determine the activation of lower layer-coordination control layer, and provide parameters for correct planning and execution. In the lowest level of the architecture, the coordination control layer includes controllers for imposing vehicle speed profiles, implementing cooperative adaptive cruise control methods (platooning, and planning/executing merging and leaving maneuvers). The overall architecture of the cloud platform is consisted of 3 layers: CARMA vehicle, CARMA edge, and CARMA core cloud. From Ref. [26], the framework of the CARMA is evolved to have CARMA 3rd party services interacting with its core cloud. Its on-board vehicle network connects various on-board sensors, infotainment equipment, on-board embedded processors, HMI equipment, and actuators to apply control commands. The onboard control components operate in cooperation with the edge controller assuring fault-tolerance of the system in cases when the connection with the CARMA Edge is disrupted. Further, since vehicle safety is of paramount importance, on-board controllers are also responsible to assess and potentially override the remotely computed instructions (from cloud/edge) to ensure safety of the vehicle. The CARMA Edge sub-system hosts off-board processes and information that require tight access (low latency) with the vehicles. This will include information collected from the vehicle and the processes that require cooperation with roadside equipment and other vehicles. The CARMA core cloud is the highest level of cloud platform layer that acquires environment data from 3rd party, as well as vehicle ID information with corresponding data, including vehicle states, positions, platoon information, etc. CARMA Edges and other modules are implemented within the CARMA Core to calculate optimal speed profile, inter-vehicle distance, and commands to CARMA Edge. The CARMA project initialized the concept of cloud platform with a core cloud controlling edge clouds built in each road network segments for vehicle platoon control. Then, the edge cloud gives vehicle commands, which forms the basic structure of cloud control platform.

In Ref. [27], Hussain et al. mentioned a vehicular cloud based on vehicular ad-hoc network (VANET) to define the potential architectural framework of this type of cloud platform. The architecture is divided into three frameworks: vehicular clouds (VC), vehicles using clouds $(\mathrm{VuC})$, and hybrid vehicular clouds (HVC). From the hierarchical level, the system is divided into three layers: car, inter-car, and cloud levels. The bottom level is the communication at car level. Vehicle in standalone VANET has Global Positioning System (GPS) to obtain accurate location information, radar, sensors, and actuators. The second level of the communication is intercar level where vehicles communicate with each other via On-Board Unit (OBU). This communication can be either V2V or V2I by using IEEE 802.11p (WAVE) standard. The top most level enables vehicles to communicate at cloud level where vehicles or RSUs may serve as gateways. The $\mathrm{VC}$ is further divided into two scenarios (static, dynamic) from movement standpoint. The static clouds refer to the stationary vehicles providing cloud services (renting out storage or processing resources). In case of state VANET clouds, the infrastructure can be rented out to make revenue as well. The $\mathrm{VuC}$ connects the VANET to traditional clouds where VANET users can use cloud services (remote configuration and car performance checking, big traffic data analysis, smart location-based advertisements, vehicle witnesses) on the move such as infotainment, traffic information, etc. In HVC, vehicular clouds interact with traditional cloud for services exchange.

From the examples, it can be concluded that vehicular clouds are commonly divided into three major layers: vehicle, inter-vehicle, and cloud levels. With the requirement of data grouping and processing, multi-access edge computing (MEC) concept has been added for local data processing and traffic control. MEC also communicates with higher level cloud layer when necessary, which reduces the load of higher layer cloud, as well as improving system efficiency by doing ICV control and data processing locally. Lots of cloud systems are still vertical cloud system, which means on each layer, the server is scaled up with memory and storage. Such concept is limited in computational ability due to the limitation of existing CPU capability. Therefore, the coverage of each cloud system is small. The development of horizontal cloud, which scales up number of servers for local computing in different areas, can greatly share the workload of central cloud and extend the coverage of a central cloud system. However, the construction of horizontal cloud requires building complete and small cloud in each divided area, which will further increase cost for area based intelligent traffic infrastructure construction. The determination about the way to develop cloud system based on traffic environment requirement becomes a difficulty in cloud implementation.

In vehicle-road-cloud wireless communication, moving vehicles need to establish a dynamic network connection quickly and reliably with communication infrastructures (such as a cellular base station), and establish a reliable wireless communication link with 
an edge cloud platform during fast driving. Currently, the traditional CAN bus on the vehicle side is gradually upgraded to vehicle Ethernet. Roadside facilities are gradually adopting high-bandwidth connections, such as Gigabit Ethernet with PoE capability. Therefore, the bandwidth is no longer a major limitation. However, due to the unstable wireless link connection from the car-side network (which is based on C-V2X or DSRC), a high-reliability and low-latency communication channel between moving vehicle and the edge cloud cannot be formed, which prevents the edge cloud platform from supporting some safety applications with extremely low latency requirement. It is necessary to set low-latency communication links for vehicle Ethernet, roadside facility and edge cloud networks to achieve reliable dynamic network. However, finding a way to design ultra reliable and low latency vehicle to cloud (V2C) and vehicle to roadside infrastructure (V2I) communication transmission mechanisms is the core problem that needs to be solved.

Funded by the Innovate UK, the i-Motor project has developed a cloud-based data platform that helps autonomous and human-driven vehicles connect and communicate with each other and their surroundings to reduce collisions and traffic congestion. This 2-year project has produced a mobile platform for data transfer and storage by vehicles from different manufacturers. The Vehicle Cloud Computing (VCC) system can securely handle 'big data' with near real-time results, which will be essential if lots of vehicles are 'talking' to each other and sharing information with traffic control centers and smart city infrastructure. The VCC can accept data from a variety of external sources and capture information from multiple vehicles to provide driver and car with timely and accurate updates on road works, congestion, weather conditions and other issues that might affect travel. The platform also allows vehicles to automatically report and self-diagnose problems to reduce the chance of a roadside breakdown or detect hazardous conditions and warn other nearby vehicles [28].

In order to improve the safety of winter driving on icy road, the Swedish Transport Administration and the Norwegian Public Roads Administration together launched a major Scandinavian Cooperative ITS (C-ITS) project for sharing road condition information via a cloud-based system. The project enables ICV to share information about conditions relating to road friction, such as rain, snow, and icy patches, through a cloud-based network. Not limited to the communication between the ICVs, the road condition alert system also sends information about icy patches to road administrators, as a complement to existing road-weather measurement stations along the road. The data will help road administrators and their contractors to better plan and execute winter road maintenance and quickly address changes in road conditions [29].

The Germany's Federal Ministry for Economic Affairs and Energy initiated a project named the Kooperatives hochautomatisiertes Fahren (Ko-HAF), to increase the safety and efficiency on the road by means of cooperative and highly automated driving at speeds up to 130 $\mathrm{km} / \mathrm{h}$ in 2015. This project aims to develop new systems and functions allowing for highly automated driving at higher speeds and in more complex situations. The edge cloud computing technology is adopted for environment recording and representation, vehicle localization, and providing cooperative driving guidance [30]. The KoHAF project supported by BMWi [22] uses information communication between vehicle and safety server (cloud) to realize autonomous driving. The safety server contains high definition map (HD Map) and obstacles on the road. The vehicle will send both static (lane, signs) and dynamic (vehicles, pedestrians) data to server. Both the server and the vehicle data form together as a cooperative environment perception data source to optimize the HD map on the server by learning algorithms. The vehicle side will download optimized map and incorporate with data from vehicle sensors to build a scenario model. Thus, the data fusion between vehicle and server can help vehicle identifying dangerous situation and initiate solution in advance, which improves the reliability of autonomous driving.

As more and more ICV projects related to cloud computing are arisen, their application field widened from intelligent driving and V2X to ITS and smart city as shown in Table 1. And ICV cloud control concept and the design methodology of its complicated system architecture is becoming the interest of the academic and industry research.

\subsection{ICV Cloud Control Concepts}

At the beginning of the century, the concept of vehicular cloud starts from the idea of mobile Ad-hoc Networks (MANET) to roadway and street communication. The original motivation for the interest in MANET was induced by the concerns such as traffic delays and congestion, to inform drivers of actual or imminent road conditions, and hazardous driving conditions, etc. Therefore, most MANET applications focus on traffic status report, collision avoidance, emergency alerts, cooperative driving and other similar concerns [31]. The Crash Avoidance Metrics Partnership (CAMP) and the Vehicle Safety Communications Consortium (VSCC) have introduced an inter-vehicle communication mobile Ad-hoc network [32]. The application target is to simulate extended brake lights (EBL) scenario in its local inter-vehicle cloud. It will 
Table 1 Comparison of different cloud related ICV projects

\begin{tabular}{|c|c|c|c|c|c|c|}
\hline \multirow[t]{2}{*}{ Project name } & \multirow[t]{2}{*}{ Area } & \multicolumn{4}{|l|}{ Application } & \multirow[t]{2}{*}{ Literatures } \\
\hline & & $\begin{array}{l}\text { Intelligent } \\
\text { driving }\end{array}$ & V2X & ITS & Smart city & \\
\hline Strategy for Innovation and Development of Intelligent Vehicles & China & $\sqrt{ }$ & $\sqrt{ }$ & $\sqrt{ }$ & $\sqrt{ }$ & [21] \\
\hline Cloud-Assisted Real-time Methods for Autonomy (CARMA) & USA & $\sqrt{ }$ & $\sqrt{ }$ & & & {$[25,26]$} \\
\hline i-Motors & UK & $\sqrt{ }$ & $\sqrt{ }$ & $\sqrt{ }$ & & {$[28]$} \\
\hline Cooperative Intelligent Transport Systems (C-ITS) Project & EU & $\sqrt{ }$ & $\sqrt{ }$ & & & [29] \\
\hline Kooperatives Hochautomatisiertes Fahren (Ko-HAF) & Germany & $\sqrt{ }$ & $\sqrt{ }$ & & & {$[30,33]$} \\
\hline
\end{tabular}

establish vehicle route only on demand based on Ad-hoc On-demand Distance Vector (AODV) routing algorithm. However, a larger and more complex vehicular configuration should be added to more closely mimic that of actual traffic.

With the deployment of energy-sufficient vehicles with permanent Internet presence, featuring substantial on-board computational, storage and sensing capabilities, in 2017, the concept of autonomous vehicular cloud (AVC) is formed to be a group of highly autonomous vehicles that corporate computing, sensing, communication and physical resources can be coordinated and dynamically allocated to authorized users. Based on cloud and vehicle side data fusion and cooperative planning, AVC can be used for different traffic management scenarios, including synchronizing traffic lights after clearing an accident, autonomous mitigation of recurring congestion, sharing on-road safety messages and the asset management control etc., mainly focusing on building a safe and efficient autonomous driving environment [31].

From previous concepts on cloud control, MANET targets to traffic status communication vehicles, and AVC targets to the sharing of dynamic high definition map between vehicles. Both of them lack of considering connected vehicles and integrating the vehicles, roads and cloud in a whole as an infrastructure. While in 2020, the concept of ICV Cloud control system (CCS) based on the integrated technology of vehicle-roadcloud and dynamic resource scheduling technology was firstly introduced systematically [13, 34]. It points out that CCS has five features: ubiquitous interconnection of vehicles, roads and clouds, digital mapping of all traffic elements, global performance optimization, efficient computing and scheduling, and high reliability of system operation. ICV CCS has been classified as the potential solution for supporting fully automated driving.

\section{Architecture Design of ICV Cloud Control System}

The rapid development of computing, communication and control technologies has led to tremendous changes in people social life. With the in-depth integration of informatization and industrialization, traditional singlepoint technology can't adapt to the new generation production equipment communication demand. In such a situation, CPS emerged as the current frontier research direction in the automation field with preliminary progress. Figure 1 shows a general cyber physical system architecture.

CPS supports the in-depth integration of informatization and industrialization. Through the integration of advanced sensing, computing, communication, control and other information and automatic control technologies, it builds the mutual mapping of human-machineenvironment communication network in the physical and information spaces. CPS is a complex system with timely interaction and efficient coordination to realize ondemand response, rapid iteration, and dynamic optimization of resource allocation as well as operations in it. The

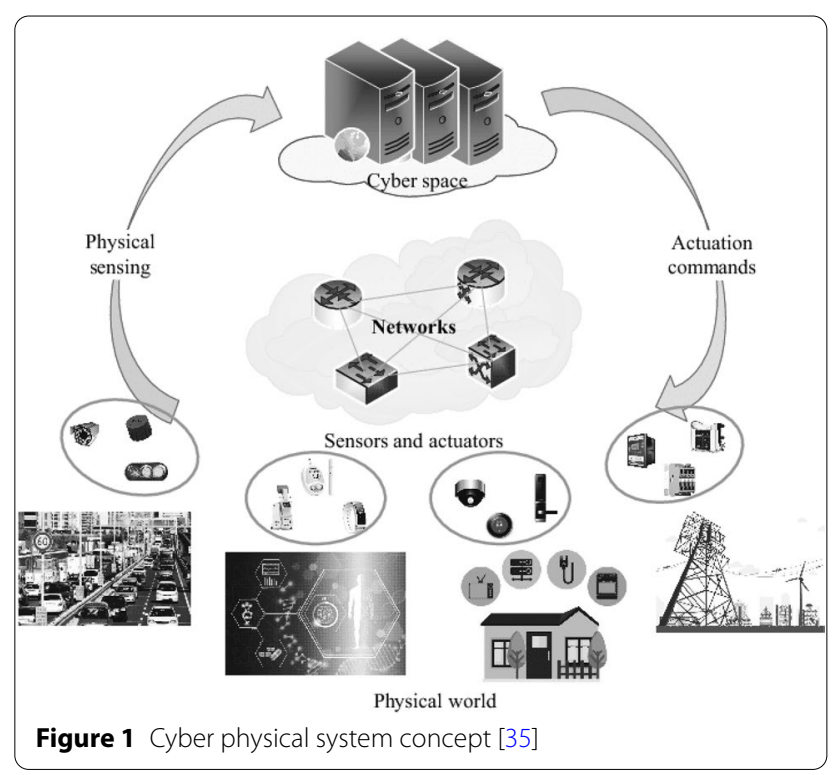


implementation of CPS is hierarchical and can be divided into three levels, that is, unit level, system level, and system of system level [35]. It consists of four core technology modules: perception and automatic control module, industrial software module, industrial network module and industrial cloud module. Currently, the research on CPS in the transportation field is mostly in the exploratory phase. In 2008, NSF in Unite States and other academic organizations organized a seminar on CPS in the transportation system, which spurred widespread attention on CPS in the transportation field. Figure 2 shows an industrial IoT solution layered with perception level, network level, and application level in cloud to illustrate a typical CPS application in the industry [36].

In terms of equipment computing capabilities, Ref. [37] points out that with the increase number of miniaturized equipment and improving computing capabilities, it is possible to achieve urban-scale traffic detection from the perspective of computing equipment. Ref. [38] shows that in all intelligent transportation systems (ITS), improving vehicle performance and reducing fuel consumption require more attention. The CPS technology becomes a possible solution. Ref. [39] points out that CPS plays an important role in supervision and safety control functions of the ITS system, which will help the actual control decision-making in the transportation system.

In terms of the structure and composition of the ITS systems, Ref. [40] believes that the CPS architecture for the transportation system should include interaction process control among software, communication network and physical devices. Ref. [41] proposes that CPS includes two important components, namely physical processes and network systems. The network system is composed of some micro-devices with perception, calculation, and communication capabilities. The typical physical process is monitored and controlled by the network system. Ref. [42] points out that as a typical application of

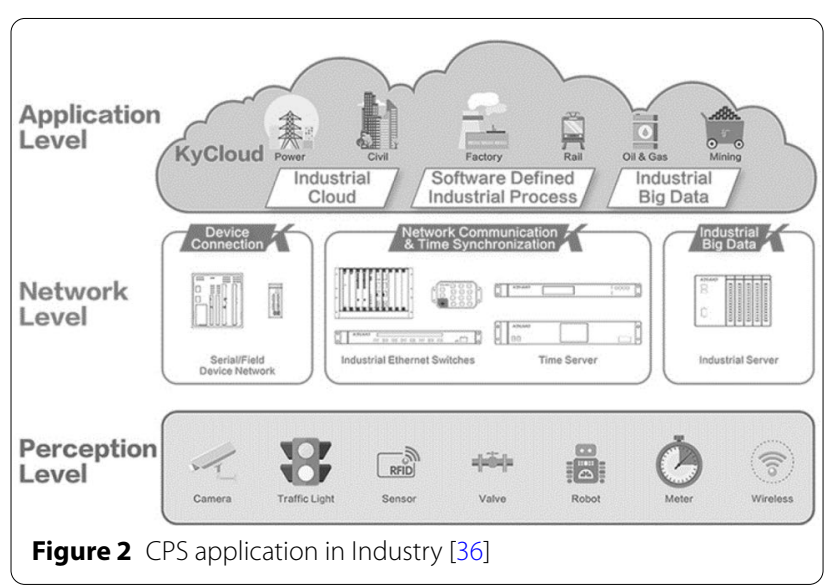

CPS, ITS system includes physical and network components. The function of physical components is to provide physical interoperability between different transportation modes, including some physical equipment such as data collection devices. The network component aspect refers to the integration of traffic information based on network communication.

To improve the scalability of ITS application scenarios, Ref. [43] believes that CPS provides the possibility for the improvement of the new generation transportation systems. However, it also brings some challenges, including the unclear system division, terminology system shortage and the lack of system reliability design and safety analysis methods. Ref. [44] shows that the modern transportation system is a typical CPS. The technical bottlenecks faced by transportation system development include reliability, reusability, and cost problems. Ref. [45] demonstrates that the current road traffic control system is not an Internet-based system, which requires a more open control method. Ref. [46] believes that current stage is the early stage of CPS development. It must have characteristics of high credibility, time predictability and robustness which requires high system scalability and refined design process. Ref. [47] elaborates on the challenges faced by the automotive control software research in CPS. It believes that accurate vehicle micro-control can reduce the energy consumption. Software control is the major way to realize vehicle micro-control. Ref. [48] proposes a CPS middleware framework for the traffic state monitoring system, including sensor monitoring nodes that can move in the area and a computing framework that provides adaptive load balancing. The framework tries to achieve system reliability, accuracy, and effective automatic control.

In China, China Industry Innovation Alliance for the Intelligent and Connected Vehicles (CAICV) proposed a CPS reference architecture for ICV [49], advised to use model based system engineering technologies and tools [50]. The latest ICV CCS architecture is also proposed by Chinese academic and ICV industry with broad consensus [34]. They clarified the ICV cloud control system's main constituents like connected vehicles and other traffic participants, road side infrastructures, basic cloud control platforms, related 3rd party supporting platforms, V2X network communication links and cloud application platforms etc. The structure can be concluded into Figure 3 [13].

This architecture is recognized as the most promising solution for ICV cloud control system implementation due to the strength of Chinese government overall plan on vehicle provider, road infrastructure and cloud resource coordination. Traditional V2X mainly communicates with the vehicle and the road side infrastructure, 


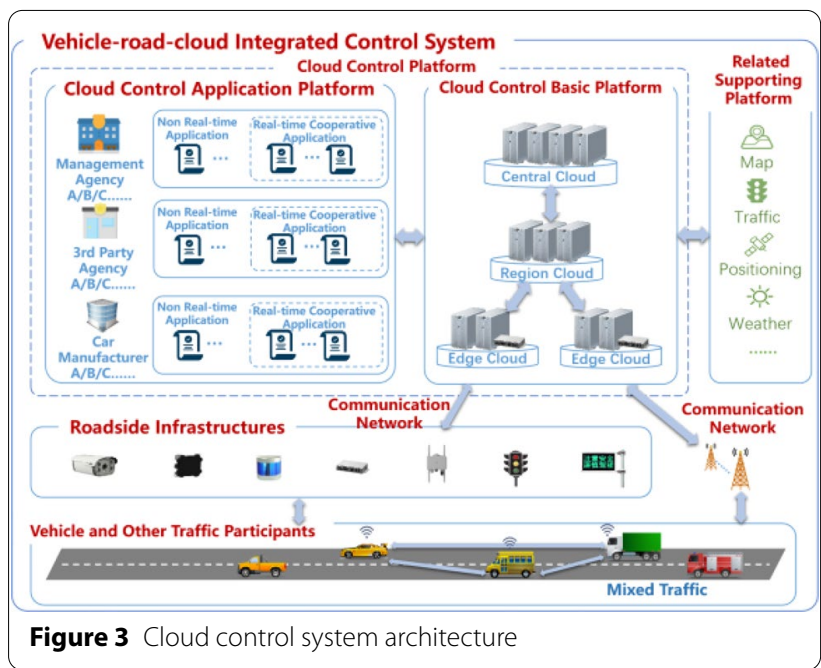

and its decision-making task is mainly based on reactive mode, while the CCS integrates it into a centralized-distributed layered system with the ability to handle widerange decision-making tasks in deliberate mode.

In this section, the concept of CPS has been introduced. The CPS is a solution to satisfy new generation manufacturing demands according with in-depth integration of informatization and industrialization. Then, the importance to use CPS design methodology in ITS system is identified followed by some information about the structure of the CPS architecture in the ITS system. The section analyzes the requirement of specific CPS design for the ITS system that is not only a networkbased system by providing some preliminary works. It is foreseeable that the CPS design methodology will play a critical role in ICV CCS design processes.

\section{Technologies of ICV Cloud Control System}

According to ICV CCS white paper published by CAICV [34], as a complicated CPS, ICV CCS requires kinds of technologies to be developed simultaneously. These technologies can be divided into two parts. One is ICV CCS core technologies, which are common and critical, including edge cloud and dynamic resource scheduling, fusion perception and localization, and vehicle control. The other is ICV CCS supportive technologies, including V2X communication, high definition map and scenarios library.

\subsection{Core Technologies}

\subsubsection{Edge Cloud \& Dynamic Resource Scheduling}

(1) Edge Cloud
Edge cloud for ICV is one of the emerging technologies in communication network [51] and one of common core technologies in ICV CCS. ICV CCS edge cloud is usually built on MEC(Multiaccess Edge Computing) server. As C-V2X (cellular network-based vehicle to everything) networking technology is becoming one of key technologies in many regions, it strongly supports the edge cloud architecture of ICVs based on MEC. The concept of MEC first appeared in 2013 and was originally called Mobile Edge Computing. It migrated the cloud computing platform from within the mobile core network to mobile access network. After 2016, Mobile Edge Computing service has been further extended from mobile cellular networks to other access networks with the support of more scenarios, such as V2X communications. The concept of edge cloud and C-V2X integration is to deploy $\mathrm{C}-\mathrm{V} 2 \mathrm{X}$ services on the MEC-based edge cloud platform with the help of Uu or PC5 interfaces to support "pedestrian-vehicle-road-cloud" collaborative interaction. It can reduce end-to-end data transmission delays and network load caused by massive data return. Simultaneously, it also relieves the computing and storage pressure of terminals or roadside intelligent facilities, and provides highquality services for ICVs with local characteristics.

The standardization work on MEC is mainly focused on ETSI and 3GPP. At the same time, 5G Automotive Association (5GAA) also conducted in-depth discussions on C-V2X-oriented MEC. Among them, ETSI's MEC group focuses on MEC platform, MEC platform-based network construction, and MEC platform-based business application operation deployment. While the MEC solution proposed by 5GAA is based on the one from ETSI, 3GPP mainly studies the network capabilities that 5G network architecture needs to support MEC and integrates $\mathrm{MEC}$ and $\mathrm{C}-\mathrm{V} 2 \mathrm{X}$ as a recommended solution.

(2) Dynamic Resource Scheduling

For dynamic resource scheduling, the main process is to manage resource, which is very important in MEC systems. The joint management of radio and computing resources plays a key role in achieving energy-saving and low-latency MEC. The network architecture in which the MEC server and wireless AP (Access Point) are co-located, helps to realize related technologies. A comprehensive overview of the MEC system resource management literature is carried out. Our discussion started with a simple single-user system consisting of a mobile device and 
a MEC server. Subsequently, a more complex multiuser MEC system was considered, in which multiple offloaded users compete for the use of radio and server computing resources, and coordination has been conducted. Finally, MEC systems with heterogeneous servers, which not only provide freedom of server choice, but also allow collaboration between servers are depicted. Such network-level operation can significantly enhance the performance of the MEC system.

For single-user MEC systems, binary offloading, partial offloading and stochastic models are common task models used. Ref. [52] designs the optimal threshold of resource allocation based on the optimal offload function of Time Division Multiple Access (TDMA) and Orthogonal Frequency Division Multiplexing Access (OFDMA) systems. Ref. [53] proposes a new wireless-powered MEC framework that can simultaneously optimize local computing and offloading. Ref. [54] proposes a general standard for the offloading decision of energy consumption, computing minimization, and delay reduction. Ref. [55] provides a joint scheduling and computational offloading algorithm for parallel processing through appropriate parallel processing components on the mobile terminal and the cloud. Ref. [56] is based on the Lyapunov optimization algorithm to determine the offloading strategy, the CPU clock speed during task execution and the selected network interface. For multi-user MEC systems, joint radio-and-computational resource allocation methods, MEC server scheduling and cooperative computing models are common topics that researches are interested in. Ref. [57] uses an iterative algorithm to solve the problem of C-RAN's non-convex resource allocation. In order to realize ubiquitous edge computing, heterogeneous MEC system was proposed in Ref. [58], including a central cloud and multiple edge servers. Coordination and multi-layer central/ edge cloud interaction introduces many new research challenges and have recently attracted a wide range of further research. Ref. [59] proposes a distributed resource allocation algorithm based on Nash equilibrium. Ref. [60] constructs a congestion game and proposes a distributed algorithm considering Nash equilibrium. Ref. [61] carries out the research on the distributed algorithm and active caching algorithm of cooperative tasks in MEC.

In the MEC and C-V2X converged system, the MEC platform can orchestrate IT basic resources for Internet of Vehicle (IoV) edge applications on demand, and configure computing and storage capabilities. In the mean time, it can provide a virtualized application hosting environment that can manage the life cycle of edge cloud and monitor applications as well. Besides, it can also distribute general network and IoV business information and other information for applications. According to the C-V2X communication mode used by the terminal to access the MEC platform, the MEC and C-V2X converged system can be divided into two types, that is, Uu MEC and PC5 MEC. When the MEC platform is deployed in the operator's network, the terminal accesses through the Uu interface. In this case of the MEC platform, it is defined as a Uu MEC; When the MEC platform is relatively independent of the operator's network, with the terminal accessing the RSU (road side unit) through the PC5 interface followed by MEC platform accessing, it is defined as a PC5 MEC.

\subsubsection{Fusion Perception \& Spatial and Temporal Localization}

Fusion perception and localization is another common core technology in ICV CCS.

(1) Fusion Perception

For long time, visual perception is the main approach in the ICV domain $[62,63]$. Especially, in recent decades, with the development of deep learning [64], visual perception has promoted the wide application of autonomous driving technology [65]. Visual perception includes the detection of traffic participants, lanes, obstacles, traffic signs, traffic signals, and derivable areas within the camera scope [66-68]. In ICV CCS, the visual perception can be divided into two categories based on whether it is vehicle side or road side cameras. As there is a great difference between the camera scopes of ICV and road side infrastructure, visual detectors are influenced by both the camera scope and the related data set. Currently, the opensource data set like KITTI [69] and ApolloScape [70] are mainly from the vehicle side. Data sets based on sensors of road side infrastructure are attracting more attention. For instance, VERI-Wild [71] is a typical Chinese transportation data set for ReID published by Pengcheng National Laboratory in China. However, big data sets are still required to depict different transportation environments in different countries to improve detectors accuracy. Besides, the method to obtain abnormal vehicle data set is still an ongoing problem without any solution. Thus, the data acquisition problem blocks the way of fast and accurate perception in real scale applications. 
Starting from 2012 when AlexNet Neural Network had been introduced [72], several detectors have been developed, including two-stage detectors like R-CNN (regionbased convolution neural network), R-FCN (region-based fully convolution network), Fast RCNN, Faster RCNN and Cascade RCNN, and one-stage detectors like YOLO (you look only once), SSD (single shot multibox detector), and RetinaNet, etc. In Ref. [73] YOLOv4416 can process 100 images per second, which is faster than previous deep networks, but it requires the image quality downgrade that decreases detection accuracy especially from road side camera. From the existing literature, current visual detection algorithms can identify most kinds of objects appeared on ordinary traffic road, but are still greatly affected by the external environment, especially abnormal weather that will cause system malfunction and error. Besides, small object detection is an important reference for environment prediction. Current perception accuracy on small object is not high enough, as shown in Table 2. The $\mathrm{AP}_{50}$ value of the algorithm on the MSCOCO [74] data set is around 2 to 5 times $[75,76]$ compared to the $\mathrm{AP}_{\mathrm{S}}$ value, which requires improvement.

Table 2 Detector comparison on COCO'07 datasets

\begin{tabular}{lllll}
\hline Algorithm & $\mathbf{A P}_{\mathbf{5 0}}$ & $\mathbf{A P}_{\mathbf{S}}$ & $\mathbf{A P}_{\mathbf{L}}$ & FPS \\
\hline YOLOv4608 & 65.7 & 26.7 & 53.3 & 65 \\
YOLOv4416 & 62.8 & 20.4 & 56.0 & 96 \\
YOLOv3608 & 57.9 & 18.3 & 41.9 & 50 \\
Faster-RCNN & 42.7 & - & - & - \\
Mask-RCNN & 62.3 & 22.1 & 51.2 & - \\
SSD513 & 50.4 & 10.2 & 49.8 & - \\
RetinaNet800 & 59.1 & 22.8 & 50.2 & - \\
R-FCN & 51.9 & 10.8 & 45 & - \\
\hline
\end{tabular}

In Ref. [77], Sivaraman and Trivedi reviewed the representative works in vision-based vehicle detection and tracking in detail, appending with the comparison of onroad behavior analysis methods.

Although visual perception technology has achieved gratifying results, it still has safety issues in adverse conditions, such as low light, haze, and fog, etc. The fusion perception technology is applied to improve object detection accuracy in such circumstances. Indeed, nowadays, a road side infrastructure tends to include multiheterogeneous sensors, such as camera, millimeter-wave radar (MWR), Light Detection and Ranging (LiDAR), and global navigation satellite system (GNSS) within a single system [78]. With the multi-sensor fusion perception methods [79], range and vision sensors can be combined to recognize traffic environment of both static obstacles and dynamic objects on the road, while GNSS can be used to estimate the vehicle position. Among range sensors, LiDAR mainly uses time of flight (TOF) to complete the velocity and range measurement. It has many advantages, such as accurately obtaining target's 3D information, high resolution, strong anti-jamming ability, wide detection range and near all-weather operation. For intelligent vehicles, LiDAR is a necessary sensor [80-82]. Recently, it is also applied on roadside to detect not only static information such as road lanes [83], but also dynamic environment, including pedestrians, cars and buses [84-86] to better serve connected vehicles. MWR is another kind of range sensor, which is commonly used on both vehicle and road sides. Depending on the frequency band, $77 \mathrm{GHz}$ radar is widely equipped on the vehicle side [87]. While on roadside, not all kinds of MWR can be allowed to be deployed. Pre-crash safety warning is a well-known application for vehicle side radar application [88]. Table 3 shows different

Table 3 Comparison of different sensors

\begin{tabular}{|c|c|c|c|c|c|c|c|c|}
\hline Category & Sub-category & Adaptability & Scope (m) & Accuracy & $\begin{array}{l}\text { Recognition } \\
\text { ability }\end{array}$ & $\begin{array}{l}\text { Range } \\
\text { measurement }\end{array}$ & $\begin{array}{l}\text { Velocity } \\
\text { measurement }\end{array}$ & $\begin{array}{l}\text { Vehicle/ } \\
\text { road } \\
\text { side }\end{array}$ \\
\hline Visual & Monocular [93-95] & * & $80-120$ & * & * & * & * & $y / y$ \\
\hline Visual & Stereo $[95,96]$ & * & $\leq 50$ & $* * *$ & $* * *$ & * & * & $y / y$ \\
\hline Visual & Panoramic $[97,98]$ & * & $200-250$ & * & N/A & N/A & N/A & $y / y$ \\
\hline Visual & Infrared $[99,100]$ & $* * *$ & $150-300$ & $* * *$ & $* * *$ & N/A & N/A & $y / y$ \\
\hline Radar & MW Radar $[85,101,102]$ & $* * *$ & $\begin{array}{l}5-70(24 \mathrm{GHz}) \\
150-200(77 \mathrm{GHz})\end{array}$ & * & $* * *$ & * & * & $y / y$ \\
\hline LiDAR & 4-line $[103,104]$ & $* * *$ & $0.3-200$ & * & $* * *$ & * & * & $y / y$ \\
\hline LiDAR & 16-line $[105,106]$ & $* * *$ & $0.3-200$ & $* * *$ & $* * *$ & * & * & $y / y$ \\
\hline LiDAR & 32 -line $[106,107]$ & $* * *$ & $0.3-200$ & $* * *$ & $* * *$ & ${ }^{*}$, Height & * & $y / n$ \\
\hline LiDAR & 64 -line $[106,108]$ & $* * *$ & $0.3-200$ & $* * *$ & $* * *$ & ${ }^{*}$, Height & * & $y / n$ \\
\hline
\end{tabular}

Note: ${ }^{* \prime}$ for weak/low, ${ }^{\prime * * * \prime}$ for strong/high, 'y/n' for yes/no and 'N/A' for not applicable 
sensors' characteristics in terms of environment adaptability, sensing scope, detection accuracy, object recognition ability, range and velocity measurement, as well as whether vehicle or road side product is ready to be used.

In Ref. [89], researchers summarized the roadside sensors related works in detail, and pointed out that the monocular vision sensor is still the main category setup on the roadside. In Refs. [90, 91], the fusion perception methods of LiDAR and camera data are proposed to increase single sensor accuracy. In Ref. [92], fusion perception of radar and camera of vehicle side is proposed. Existing fusion perception methods can significantly improve the accuracy of the object detection and enhance the object tracking ability on the road. Most of them can only focus on target level but seldom on feature or raw data levels. This is not only due to performance consideration, but also due to limited point cloud data acquisition of range sensors. In the ICV CCS, the fusion perception on both vehicle and road needs to have good adaptability, robustness and highly usability, in order to acquire real-time, high-precision, and high-reliable basic dynamic data to meet needs of ICV autonomous driving and transportation digital twin.

(2) Spatial and Temporal Localization

A more accurate fusion perception, especially in object re-identification, can be achieved from a high quality spatial and temporal localization [109, 110], which usually can be achieved by GNSS device.

In ICV CCS, the locations of traffic participants, road side facilities, and traffic events all require reliable accuracy assurance, low latency transmission, high availability in complex scenes, security redundancy, as well as robustness. In Ref. [111], researcher emphasized the importance of spatial temporal characteristics to moving objects. With the establishment of intelligent fusion perception based on semantic characteristics combined with high-resolution maps and high-precision positioning technology, it can ensure reliability, accuracy and availability of spatial and temporal localization in various application services.

\subsubsection{Vehicle Control}

Finally, vehicle control is the most important common core technology in ICV CCS. The development of vehicle control system starts from the electrification of automobile (the development and application of ECU and mechatronic actuators). One major field of vehicle control is dynamic control, which plays crucial rule in ICV as well. Starting from the application of control unit in conventional vehicles that focused on individual vehicle, the target is to keep vehicle motion stable for safety purposes and improve ride comfort of passengers. Several control units have been developed. Vehicle motion control can be divided into longitudinal, lateral, and ride control.

(1) Vehicle control: from individual to cooperative

- Longitudinal Control

Currently, the cruise control (CC) plays an important role in longitudinal control to reduce driver workload. The first modern cruise control hardware patent was published in 1956 [112]. At the beginning, $\mathrm{CC}$ only has the capability to maintain a constant speed in an open road with gas pedal control. The control method is simply a PID feedback controller that uses the gas pedal as an actuator to minimize vehicle speed error. The sensor is just the odometer. However, the applied vehicle longitudinal model was linearized equation with the ignorance of vehicle engine system dynamics and the assumption of constant time constant and gain. In real situation, vehicle forward dynamics are nonlinear, and the corresponding linear dynamics will vary depending on the operating conditions. These issues had been addressed by various studies in the literature (e.g. in Refs. [113-115]), which introduced the development of adaptive cruise control for better robustness. An adaptive cruise control (ACC) is an automobile system which purpose is to control the velocity of the vehicle with regards to the surrounding environment (other vehicles) [116]. With the development of various sensors (radar, LiDAR, etc.) equipped on vehicle, ACC extends the function of CC by adjusting vehicle speed via environment (road conditions, weather conditions, other vehicles appearance, etc.). Car following model was developed based on a following vehicle with a leading vehicle [117]. The basic scenario of an ACC system is a car following scenario, therefore, the control of ACC focused on keeping a fixed distance in between following and leading vehicles. With the purpose to adjust the distance, car avoidance system was also incorporated with ACC by controlling vehicle braking system to allow vehicle accelerating and braking. To obtain more optimal control commands, several modern optimal control strategies have been applied, including linear quadratic regulator (LQR) [118], model predictive control (MPC) [119], or even hybrid MPC (HMPC) [120]. With the application of MPC method, prediction becomes more important since the method is based on a prediction window with the capability to adjust system 
model and handle system physical constraints. For individual vehicle, prediction depends on vehicle equipped sensors, including some supersonic radar, LiDAR, or even camera. The scan capability of such on-board equipment can be limited (from several meters to one hundred meters). A vehicle cannot have the ability to predict the overall traffic condition along a road network, which limits the performance of the controller.

- Lateral control

The lateral control unit includes electronic stability control (ESC), four wheel steering control (4WS), differential braking, etc. The purpose of lateral controller is to maintain vehicle yaw and roll stability during steering maneuvers. Currently, in most literature, with the possibility to apply linear control strategies, vehicle model is simplified into bicycle model when roll motion is not considered. With the roll motion considered, vehicle weight transfer must be considered. Thus, bicycle model cannot be appropriate anymore, which let researchers introduce more complicated model that includes roll motion as well as nonlinearity of vehicle system. The ESC, 4WS, and differential braking systems use front wheel steering input, rear wheel steering input, as well as braking torque distribution on each wheel to adjust vehicle yaw motion to keep vehicle side slip angle within controllable limit. The lateral control problem can be modeled as a path-tracking problem with the curvature of the road as system disturbance input. The goal is to control a vehicle to use lateral control actuator to follow a path without causing stability problem. Based on the tracking characteristics, lots of feedback control strategies have been developed. For example, Zhao et al. applied differential brake torque with yaw stability control for lateral control with the application of fuzzy logic controller in an intelligent vehicle highway system for lane keeping task [121]. Some other nonlinear control strategies, including chained systems theory [122], nonlinear Model Predictive Control (MPC) [123] are also applied on vehicle path-tracking control. With linear model, the Linear Quadratic Regulator (LQR) and MPC methods are also widely applied based on their real-time implementable capability. One famous application is the Apollo LQR algorithm in path-tracking for intelligent vehicle [124]. From current development of strategies, pathtracking is a core problem in autonomous driving vehicle control, several different methods borrowed from existing vehicle lateral control strategies have been re-developed; some new developed methods based on neural network or deep learning also expands the research area of classic vehicle control. Since in most cases, a path-tracking behavior includes a combination of longitudinal control and lateral control, algorithms are developed to control vehicle acceleration/deceleration as well as steering to realize path-following function. From Ref. [125], a detailed summarization of algorithm had been provided with thorough investigation. In Table 4, a simplified table according to Ref. [125], which is about the comparison among different path-tracking control methods developed in the last decade, has been specified.

From the table above, it can be concluded that simple methods will have high real-time implementable capability with low performance under undesired disturbances, while robust or stable methods will require large computational cost which blocks the way for real-time implementation. Currently, for lateral control in autonomous driving, simple methods such as Stanley, PID and $\mathrm{LQR}$ becomes the practical strategies for realvehicle implementation. However, the challenges to deal with unexpected external disturbances and computational load requirements of more robust methods still require deep research in both software and hardware perspectives.

- Ride Control

The ride control always focuses on vehicle suspension control to mitigate undesired vibration induced by road surface or sudden jerk caused by heavy acceleration or brake. There are two major targets for ride control: ride comfort and tire dynamic load reduction. The former target is to reduce vibration that makes passengers feel uncomfortable; the latter target is to improve vehicle handling performance for stable motion. Since the vehicle ride model is always modeled as a mass-damper-spring system. Several frequency domain damping control methods had been investigated. Such control methods are called as semiactive control, since damping system can only extract energy from the vibration motion. From the simplest Skyhook control with only two damping tuning stages during the compression and extension of the suspension system [156], to three stages damping tuning control by using powerdriven-damper [157], to flexibly tuned damping control strategy [158], the tuning stages of suspension greatly increased to smoothly adjust system damping based on road profile input. Besides semi-active control, active control methods that assumes suspension can have the capability to pro- 
Table 4 Summary of path tracking control methods [125]

\begin{tabular}{|c|c|c|c|c|}
\hline Control methods & Optimization objectives & Advantages & Disadvantages & References \\
\hline Pure Pursuit \& Stanley & $\begin{array}{l}\text { Position deviation \& course } \\
\text { deviation }\end{array}$ & $\begin{array}{l}\text { Simple layout, suitable for vehi- } \\
\text { cle position control }\end{array}$ & $\begin{array}{l}\text { Difficult to apply to high speed } \\
\text { and large road curvature condi- } \\
\text { tions }\end{array}$ & {$[126-128]$} \\
\hline PID & $\begin{array}{l}\text { Position deviation \& course } \\
\text { deviation }\end{array}$ & Simple, easy to apply & $\begin{array}{l}\text { Poor versatility, difficult in tuning } \\
\text { control parameters }\end{array}$ & {$[129-132]$} \\
\hline Model-Free Control & Preview course deviation & Simple controller structure & Stability analysis is more difficult & {$[133-135]$} \\
\hline LQR & System states \& control input & $\begin{array}{l}\text { Easy to achieve closed-loop } \\
\text { optimal control objective }\end{array}$ & $\begin{array}{l}\text { Controller design based on } \\
\text { linear model (poor robustness) }\end{array}$ & {$[136-138]$} \\
\hline Feedforward and Feedback & $\begin{array}{l}\text { Feedback error, feedforward } \\
\text { information }\end{array}$ & $\begin{array}{l}\text { Able to deal with external dis- } \\
\text { turbances, modeling errors, and } \\
\text { sensor noise }\end{array}$ & Require more expensive sensors & {$[139,140]$} \\
\hline MPC & System states \& control input & $\begin{array}{l}\text { Able to handle system con- } \\
\text { straints and future prediction in } \\
\text { design process }\end{array}$ & $\begin{array}{l}\text { Difficult to analyze system stabil- } \\
\text { ity, has high computational cost }\end{array}$ & {$[141-143]$} \\
\hline $\mathrm{H}_{\infty}$ Control & System $\mathrm{H}_{\infty}$ performance index & $\begin{array}{l}\text { Easy to establish } \mathrm{H}_{\infty} \text { constraints, } \\
\text { strong robustness }\end{array}$ & $\begin{array}{l}\text { Has complex solution process } \\
\text { and theoretical derivation, can } \\
\text { only handle bounded distur- } \\
\text { bances }\end{array}$ & {$[144-146]$} \\
\hline Sliding Mode Control & $\begin{array}{l}\text { Position deviation \& course } \\
\text { deviation }\end{array}$ & $\begin{array}{l}\text { Fast response and insensitiv- } \\
\text { ity to parameter changes and } \\
\text { disturbances }\end{array}$ & $\begin{array}{l}\text { Chattering effect that requires } \\
\text { adaptive mechanism }\end{array}$ & {$[147-149]$} \\
\hline Robust MPC & System states \& control input & $\begin{array}{l}\text { Able to handle system con- } \\
\text { straints and has strong robust- } \\
\text { ness }\end{array}$ & $\begin{array}{l}\text { Difficult to analyze system stabil- } \\
\text { ity and has high computational } \\
\text { cost }\end{array}$ & [150-152] \\
\hline $\begin{array}{l}\text { Neural Network-based Observa- } \\
\text { tion }\end{array}$ & $\mathrm{N} / \mathrm{a}$ & $\begin{array}{l}\text { Optimal approximation, rapid } \\
\text { training, fast convergence }\end{array}$ & $\begin{array}{l}\text { Require large amount of vehicle } \\
\text { state information for training }\end{array}$ & {$[153-155]$} \\
\hline
\end{tabular}

Note: Table 4 is a summarized table from Ref. [125] in the last decade. Please refer to Ref. [125] for the full list of path-tracking algorithm review.

duce active force have also been investigated. The most commonly applied method can be LQR and MPC methods that adjust active suspension force to reduce the root mean square value of vehicle body vertical acceleration under random excitation or even during hard braking scenario for both ride comfort and pavement protection [159].

- The necessity to build vehicle and environment network for intelligent vehicle

The individual control development for lateral, longitudinal, and ride motions cannot have good performance in real environment, since for most of the cases, motion of a vehicle is a combination of dynamic in all degree of freedoms (DOFs). For example, the steering maneuver also includes longitudinal control, as well as ride control, due to motion tracking on the longitudinal direction and the weight counteracting control through suspension force control caused by vehicle weight transfer during steering maneuver. Therefore, mixed dynamic control becomes a new direction to mitigate multi-DOF motion of vehicle dynamic model for better dynamic behavior. In 1999, Firtz et al. had mixed 2 layer longitudinal controller with a lateral controller in truck following scenario
[160]. Peng et al. designed a frequency shaped linear quadratic (FSLQ) optimal control strategies (preview controller) to permit incorporating frequency domain design specifications. Such control strategy utilizes the good tracking performance of preview control as well as frequency domain tuning for better ride comfort to mix both lateral and ride motion control [161].

In the application of autonomous vehicle control, path planning becomes a crucial part for vehicle guiding, since human is no longer in charge of driving line selection. The field of autonomous vehicle control should be extended to include path planning for higher level guiding control incorporated with lower level dynamic control. With equipped environmental sensors, autonomous vehicle may have perception capability up to hundred meters, however, weather condition and other environmental disturbances will greatly reduce perception capability. Path planning will be limited and not smooth due to low visibility and cause abrupt control command, e.g. overly aggressive steering or heavy braking to avoid suddenly appeared obstacles, which will cause poor lateral and ride comfort level. Sometimes, vehicle 
even cannot effectively implement control command and cause severe accident, just like the car accident with truck caused by wrong perception of Tesla vehicles. Besides integrated single vehicle dynamic control, interactions among multiple vehicles become a new challenge in autonomous vehicle application, which introduces a new field called cooperative control. On the control method aspect, it controls the maneuvers of multiple vehicles within a traffic scene or network. The major consideration is the interaction optimization to smooth traffic flow in a macroscopic view. The cooperative model can be modeled as an optimization problem. As pointed out in [162] summarized by [163], a general traffic control problem will contain state variables (queue length, travel time, vehicle speed, position, etc.) and environment inputs (arrival vehicles, arrival platoon, signal timing, phases, etc.). The objective is to optimize a certain performance index (mobility, fuel emissions, safety, etc.) over a finite time horizon. Performance index may also be combined. Performance index is usually mobility-based or sustainability-based objectives or the combination of the two. The decision variables (signal timing, phases, vehicle acceleration, turn, etc.) are a sequence of control inputs. Constraints include initial conditions (queue length, flow speed, etc.), traffic flow dynamics, and vehicle dynamics. Vehicle dynamic models are simplified as lower order equations related to speed and acceleration. The traffic model will be modeled with certain pattern (car following, queuing model). The optimization problem can be solved by multiple optimization methods, including dynamic programming, learning-based algorithms, nonlinear programming [164], etc. Such control problems have been investigated with some preliminary works based on different traffic scenarios from the research at Tsinghua University as well. In Refs. [165, 166], Zhen et al. and Wang et al. did an theoretical analysis to investigate the controllability, stability, and reachability of a mixed traffic environment (human-driving and autonomous-driving vehicles) by applying cooperative optimal control on autonomous vehicles. They validated autonomous vehicle's potential in smoothing traffic flow in an mixed environment. Xu et al. [167] introduced a systematic approach to the cooperation of connected vehicles at unsignalized intersections without global coordination by developing a distributed observation and control algorithm. The result shows cooperative passing of vehicles without global coordina- tion at the cost of a growth of $8.8 \%-18.1 \%$ average travel times in low and medium traffic volumes. In Ref. [168], a dynamical interlaced layered formation generation method is introduced to provide safe distance among vehicles and efficiency for coordinated lane changing and formation switching simultaneously in real time. The method could increase the traffic efficiency by utilizing maximum road capacity while decreasing travel time for all vehicles in multiple traffic scenarios.

On the implementation aspect, cooperative control task includes perception, decision making and control. Perception uses different information detected by vehicle and road to obtain instant driving related dynamic traffic data, which provides perception data for decision making process. The road side infrastructure uses fixed sensors to form multi-sensor network for sensor fusion, which has stable range and results [169]. Multivehicle cooperative perception uses information uploaded from different vehicles for sensor fusion $[170,171]$. It is suitable for road surface perception, however, with unstable detection range and require vehicle to heavily involve into the traffic. Therefore, cooperative control requires the fusion of vehicle and road side perception. The development of cooperative adaptive cruise control (CACC) is a good example in implementing cooperative control, since it optimizes platoon operation with inter-vehicle cooperation, perform highly efficient merging maneuver, as well as cooperative control between vehicles and traffic signals at intersections. Hence, cooperative control helps to solve the difficulty in directly controlling single vehicle. It controls both traffic signs and vehicles to optimize the traffic flow globally, which can be a more effective way compared to vehicle-only control.

From the control perspective, a system with prediction capability can greatly improve control performance, since controller will have future plant dynamic knowledge to pre-calculate optimal command for better performance. Meanwhile, a vehicle will be driven in an environment with the interaction of other individuals, including pedestrians, vehicles, etc. The environmental interaction acted as external disturbances can also affect control performance, which requires the knowledge of the surroundings. Human-driven vehicle can depend on the visual and hearing ability of driver to perceive environment. However, the development of controller on autonomous vehicle must require higher robustness when external disturbance 
appears, since human will not be involved in during autonomous driving mode. Thus, prediction and environment data become much more important on autonomous vehicle application. Lots of control methods, including PID, LQR, MPC, Sliding Mode Control, or even Dynamic Programming can be incorporated or must be incorporated with predicted data. At the meantime, controller development becomes complicated due to the necessity of consideration of external disturbances. From the technological development of vehicle, multiple sensors, including millimeter wave radar, LiDAR, super-sonic radar, and camera have been equipped onto autonomous vehicle to solve data requirement problems. However, the controller performance is still limited and not globally optimized due to the limited range of sensors. Therefore, the next stage is the development of cloud platform that can connect each individual within a transportation network to provide vehicle control with the purpose to globally optimize traffic flow in all directions.

(2) The concept of cloud control platform and core technological difficulties

Based on the rapid development of big data allocation, communication technology and computing capability, internet of things (IOT) becomes realizable. The internet of vehicles will have communications, storage, intelligence, and learning capabilities to anticipate customers' intentions. The concept that will help transition to the internet of vehicles is the vehicular cloud, the equivalent of internet cloud for vehicles, providing all the services required by autonomous vehicles [172].

- High speed and complete data transfer requirement

A cloud is like a top level monitor and commander that is in charge of an area. This area includes agents (vehicle, infrastructure, pedestrian, etc.) interacting with each other dynamically. The first core technology difficulty in vehicular cloud control is the communication capability. Since large number of agents requires cloud terminal to provide path-planning suggestions, the amount of instant dynamic data that need to be transferred along the cloud network will be huge. For a high efficiency vehicular cloud, data stream also needs to be regulated to identify necessary receiver to avoid unnecessary receiver acquiring data. Therefore, despite of control data, there should be another type of data that determines receiver list for each data set released from one agent. The dynamic traffic network operation can be fast, for most of the cases, seconds level time-delay may cause catastrophic accident. Then, the vehicle actuator requires time to control the vehicle based on cloud commands. Hence, the development of high speed data transfer network is necessary. Feedback control methods have been widely used in vehicle dynamic tracking control due to its robustness. However, such methods heavily rely on the competence and accuracy of vehicle dynamic states. Although, observer can be designed to estimate vehicle states when states are not available. The estimation model introduces inaccuracy as well, which will affect control performance. With this reason, cloud network must has the capability to transfer sensor data released from vehicles with high level of integrity for effective controller performance.

- Data regulation between vehicle and cloud From literature, it is obvious to see that different control method approaches require different vehicle data. Even for different controllers that will result in same control target, the data requirement may be different, since algorithm may have different control inputs and different model detail levels. With cloud control concept, control algorithms will be integrated with cloud computing, which will result in multiple types of data requirements. To achieve high efficiency, redundant or unnecessary data must be identified and prevented from receiving from the cloud side. This requires data summary of algorithms integrated in the cloud. Lots of basic data in path-planning, such as vehicle speed, vehicle acceleration will be needed by almost all control methods, the cloud should have a regulation predefined to classify basic data needed, as well as specific data required to control specific vehicles. This also requires vehicle to upload some specific data to cloud for extra data requirement. Thus, the second core problem that needs to be solved is to build up appropriate data regulation and filtering system in the cloud network.

- Determine control algorithm distribution on vehicle and cloud sides

Vehicle has its own control algorithms integrated in ECU. It has its own sensor for vehicle dynamic detection, and it's the only way the cloud can obtain the operation data, except for basic motion data (velocity, acceleration, position, etc.) that can be obtained based on environmental perception, from vehicle side. A problem may arise about the 
way to distribute control responsibility between cloud and vehicle. It is difficult to determine if cloud needs a detailed vehicle dynamic model for control command that considers vehicle response capability. From the past, according to robot pathplanning control, the agent (robot) model incorporated online is a simplified model. Such processing method is also common in traffic control, since detailed model will greatly increase the complexity of cooperative model, which is formulated on a multiple agent level and is required to describe the relationship among agents. Vehicle is treated as a mass point inside a traffic network with simple model that only contains its basic motion data. However, the basic motion data is resulted from a detailed and complicated powertrain operation including engine, transmission, and wheels. If only do path-planning based on simple model, vehicle capability to react towards control command cannot be determined, which will result in useless planning. Therefore, including detailed vehicle dynamic model on the cloud is necessary. However, heavy storage of vehicle model will increase workload of cloud. The balance between these two factors becomes another core problem. Large number of experiments need to be done to test the requirement level of vehicle model detail a cloud network needs based on the control algorithm integrated on the cloud to let cloud do correct commands.

- Cooperative control developed in cloud platform Current cooperative control methods using hard constraints to limit traffic flow to represent vehicle motion capability to keep up with traffic flow. Such simplification requires cloud to have instant access to traffic flow data. By using traffic flow historical data, intelligent algorithms, such as deep learning are used to learn the traffic flow pattern, then predicts flow behavior in the future as the reference for the cloud system to determine if the control command can be realized by current participants (vehicles) in the traffic network. This may arise another problem, traffic data can be a way to describe normal condition of a traffic flow. However, if sudden disturbance appears (e.g. an extremely fast or slow vehicle or platoon appears in the network), the learning model needs to acquire new data for training purposes.

Based on current data sharing capability along the cloud network, vehicle speed, acceleration, position and the distance between vehicles are the easiest data that can be obtained. According to vehicle VIN number, vehicle make, brand can be obtained as well. Cloud can include a database that contains vehicle basic data (maximum performance) obtained from vehicle manufacturer. Then, the cooperative control may have one more information about how fast a vehicle can react to a path planning command. This will provide a more detailed model with maximum performance as constraint to let cloud find control commands. In the cloud, it should still consider the traffic control in a macro perspective, since data load can be a core problem. The constraints of the optimization methods should be further improved to consider vehicle specific conditions. Such data can be released from vehicle, which means cloud should have authority to access some of the ECU data to know some basic internal dynamics of a vehicle. Such model can be simplified. However, an investigation about how accurate a model should be done for better control performance, as well as improve the cloud capability to handle sudden conditions. As for vehicle side, based on drive-by-wire system, the on-board CAN bus already contains different sets of operation data. Such data can be released to cloud via wireless communication. Then, cloud will not only use road-side infrastructure as a way to detect vehicle passively. Vehicle will be an active terminal to share data with cloud. In the normal driving modes that do not require extreme driving maneuvers, vehicle equipped controller can receive cloud control command with the time length an action needs to be made. Then, on-board controller will control the vehicle according to the time limitation. Currently, vehicle control strategy does not include response time as one variable that needs to be designed. Time response is always a byproduct identified based on experimental test. To achieve time limitation as a hard constraint, extended control strategy development should be addressed.

This section proposes the history of vehicle control from individual to cooperation. Then, it identifies the necessity of cooperative control in the cloud control system. Some of the works have demonstrated the effectiveness of cooperative control on ICV in enhancing traffic flow efficiency. Integrating cooperative control with cloud still arises some new challenges that require attention.

\subsection{Supportive Technologies \\ 4.2.1 V2X Communication}

The concept of the IoV is extended from the concept of Internet of Things (IoT) [173]. The IoV involves various technologies, such as wireless network communication, high performance computing, and automotive electronic and electrical architecture technologies [174, 175]. As an interdisciplinary and emerging technology, in the past few decades, it has attracted lots of research and practice in the industry and academia [176-178]. 
Throughout the world-wide IoV communication research, it is mainly divided into two major types. The first one is the IoV communication technology based on Dedicated Short Range Communication (DSRC); the second one is the IoV communication technology based on the mobile cellular network [179-181]. Since 2004, the Institute of Electrical and Electronic Engineers (IEEE) formulated the IEEE 802.11p standard for wireless access for the road environment [182]. Combined with the WAVE architecture in the IEEE 1609 protocol suite, they formed the basic protocol architecture of the IoV communication, which is widely accepted by the industry. This protocol basically achieves the goal of V2X communication. In accordance with the expected demand and industry development, the US Department of Transportation has issued a plan that requires the launch of DSRC-supported vehicles in 2021. The plan announced that all vehicles manufactured in the United States must support the DSRC standard by 2024 .

With the rapid development and evolution of cellular communications, the industry has begun to focus on researching C-V2X technology [183, 184]. In 2016, the 3rd Generation Partnerships Project (3rd Generation Partnerships Project, 3GPP) customized a dedicated protocol LTE-V2X for V2X communication in the R14 version [185], which can better support the existing IoV business. While in the beginning, the $802.11 \mathrm{p}$ based V2X is deployed on a small scale in EU, US and Japan, C-V2X is obtaining more and more momentum especially in other regions (e.g. China). In table 5, a brief comparison between DSRC and C-V2X according to Ref. [186] has been specified.

$\mathrm{V} 2 \mathrm{X}$ is the key to deliver the more predictable driving experience. It will bring significant value to individual vehicle control and can be considered as an additional sensor, which provides $360^{\circ}$ non-line-of-sight (NLOS) awareness about both environment conditions and traffic situations. However, for ICV CCS, V2X is more than just a sensor but also a communication pipeline to exchange data between vehicles and cloud control platform. Moreover, in order to achieve a fully coordinated driving experience, more data is required to be exchanged anytime between cloud control platform and densely distributed nodes in the future. The current V2X technologies will face several new challenges in this situation, such as extreme throughput requirement, edgeless connectivity, lower end-to-end latency, and higher reliability with larger failure tolerance [187]. To overcome these challenges, advanced antenna techniques, more flexible ways of connect, multi-connectivity mechanism and new frame structure design need to be investigated.

\subsubsection{High Definition Map}

With the purpose to connect data with real environment to realize system functionality, the cloud control platform requires the support of HD map. Comparing with traditional digital map, the HD Map is a type of digital road map with accuracy higher than centimeter level to fulfill the safety requirement of autonomous vehicles $[188$, 189].

The HD dynamic map base platform acquires vehicle and roadside dynamic data from cloud control and other platforms to update static map and generate dynamic map. The purpose is to realize real-time HD map requirement of ICV. In the meantime, the static map update and dynamic map generation tasks require service transportation via region and edge clouds in cloud control platform to ICV.

The HD dynamic map base platform can provide HD map base data for cloud control platform for multisource data fusion to realize ICV beyond visual range perception and vehicle-road cooperation.

The data type of HD map can be classified into two types. One is road basic data, which include lane, lane type, lane width, lane slope, and curvature. Another is the static object data on the roadside environment, such as

Table 5 The differences between of DSRC and C-V2X [186]

\begin{tabular}{lll}
\hline Topic & DSRC 802.11p & C-V2X Rel. 14/15 \\
\hline Origin & WiFi & LTE uplink \\
Roadmap & 802.11: targets interoperability with 802.11p & C-V2X Rel. 16 based on NR(5G). Operates in a different channel than Rel. 14/15 \\
Deployment & Since 2017. Mass market in 2019 & Initial deployment in 2021 \\
Cellular Connectivity & Self managed & Cellular operators can optionally apply theoretical real-time control for higher \\
& & network utilization \\
Modulation & OFDM & SC-FDM \\
Concurrent Transmissions & No & Yes \\
Line Coding & Convolution code & Turbo code \\
Transmission Scheduling & CSMA & Semi-persistent sensing of least occupied resource \\
Time Synchronization & Loose Asynchronous & Very tight Synchronous requirements \\
\hline
\end{tabular}


traffic signs, signals, obstacles, and other detailed information. The road basic and roadside environment data need to be coded to make the navigation system locate the spatial structure of terrain, road surface, objects, and the location of driving signs. Information mentioned above will be processed and shared on the cloud control platform. The cloud control platform will provide vehicle lane level perception, path planning, and real-time/static services to ICV via processed map data combined with vehicle side pose data and road side dynamic perception data.

Cloud control platform can acquire HD map data in opendrive [190], nds and KIVI format. Combined with road network traffic data, it can support real-time navigation service in high definition.

With HD map, ICV CCS can serve better for the connected vehicles in adverse weather conditions like fog, haze or rain, even with the low performance of fusion perception. HD map can also help to improve ICV pathplanning from road to lane level based on plenty of global planning algorithms [191] (such as $\mathrm{D}^{*}, \mathrm{~A}^{*}$ ), to improve the navigation accuracy.

HD map is the necessary supporting data source inside ICV CCS. It includes the sending and receiving of HD map contents. It is required to keep map data transformation accuracy during data sending and receiving to avoid data loss. In the CCS, traffic participants and time data need to be accurate. Hence, the transformation needs to be simple and accurate to keep long-term data transferring with high frequency, which will reduce data transmission delay. With the support of centimeter level positioning, ICV CCS should accurately locate accidents to inform driver or control autonomous vehicle in safe area and predict future emergencies. CCS should process vehicle and infrastructure position data more reasonably to realize effective monitoring on dynamic data. Then, it sends the combined data with centimeter level positioning data to driver. The HD positioning technology can also ensure the accuracy of global and local programming in the CCS by combing with the V2X communication [192]. The algorithms integrated in the system should be modified to be more adapted to the accurate positioning for more reasonable path planning.

\subsubsection{Scenarios}

The scenarios library for autonomous driving is a set of virtual scenarios developed based on certain test requirements. Virtual test of autonomous vehicle is extremely necessary to test functionality as well as algorithm effectiveness of vehicle on-board sensors, data transmission, and control strategies. It helps developers to identify system defects under various driving scenarios to avoid similar problems happening in real environment. Scenarios library is the core component for virtual test, since the content of the library can directly affect autonomous vehicle performance under real environment. Therefore, with effectively developed scenarios library that contains as more scenarios as possible, autonomous vehicle can be more adapted to real environment.

The scenarios library will play an important role in ICV CCS, as the traditional development process of vehicle including model development, simulation, driving simulation test, HIL test, vehicle-in-loop test, closed field test, and open road test are not enough for ICV production and a cloud control simulation with scenarios are needed.

The construction of scenarios library includes three major steps. The first step is to confirm data storage format and standards of single scenario. Currently, the standard data format that has been used internationally is OpenScenario [193]. It is primarily used to describe complex, synchronized maneuvers that involve multiple entities like vehicles, pedestrians, and other traffic participants. The standard describes vehicle maneuvers in a storyboard, which is subdivided into stories, acts, and sequences. The data is organized in a hierarchical structure and serialized in an XML file format [194]. The second step is to build single scenario that includes both static and dynamic scenarios. The static scenario construction uses object and environment data from sensors and HD maps to build spatial and visual scenario model. The dynamic scenario construction is to convert traffic sensor and road condition data into path and property data, then generate dynamic scenario. Dynamic scenario also includes vehicle dynamic models generated from ADAMS, Simpack, Modelica, etc. The third step is to build the scenarios library based on single scenario. The first sub-step is to identify the characteristics of static and dynamic scenarios, then choose suitable scenarios based on test requirements. The second sub step is to save the scenarios into the library and build search content based on test requirements [195].

Scenarios library helps to systematically classify core application scenarios in CCS, which enables the development of custom modules to solve specific problems. Tests based on scenarios library help performance improvement of cloud control platform in control algorithm and software, physical, and network architectures. In the meantime, it also promotes the application of cloud control platform, as well as developing potential functions.

This section introduces the history of V2X communication, the elements and structure of HD map and the construction procedure of scenarios library. Then, it identifies the supportive role of these technologies in the 
ICV CCS. Some of the works have identified the evolution direction of V2X communication and HD map in order to better support ICV CCS. Moreover, the implementation of ICV CCS also requires the collaborative cooperation of these supportive technologies.

\section{Conclusions}

The paper systematically reviewed cloud control system for intelligent and connected vehicle in terms of concepts, architectures, and technologies. Based on the literature surveyed and analyzed in this paper, the cloud control system's concept was formed from plenty of cloud related intelligent and connected vehicle applications, its system architecture can be designed by implementing the cyber physical system design methodology, and its core and supportive technologies are featured with multi-discipline integration, wide-range interaction and frontier commonality.

Regarding to the current development, it can be concluded as follows.

(1) Multi-stage cloud system that includes center and edge clouds, as well as individual vehicles can be the basic structure.

(2) The cooperative control especially cloud control over autonomous vehicle can be effective in smoothing traffic flow.

(3) The design methodology based on cyber physical system is an effective way in cloud control system development.

(4) The core and supportive technologies have already been developed with preliminary works ready for cloud control system construction.

However, some challenges still block wide application of cloud control system. For instance, it is still required to build cloud control system construction and operation plan at the national level. The right to data confirmation that related to intelligent and connected vehicle is still a major problem that needs classification. On the standard side, it is still necessary to find the way to build cloud control system standard system and services methodology. On the propagation side, a method to effectively promote cloud control system application examples and an overall planning for system distribution have not been developed.

According to the challenges mentioned above, the research directions of cloud control system should be concluded here as well.

(1) It is necessary to focus on top layer design development and put the task as government policy at national level.
(2) Developing a base platform to support cloud control system for cooperative task should become the major process of system architecture development.

(3) The cooperative perception, path planning and control among vehicle, road, and cloud should become a new trend in the cloud control system technology development.

\section{Acknowledgements}

Not applicable.

\section{Authors' Contributions}

WC, XD conceived of the presented idea. KL supervised the whole paper; QW, QX and TH wrote and revised the manuscript. All authors read and approved the final manuscript.

\section{Authors' Information}

Wenbo Chu, born in 1986, is currently a Research Fellow at China Intelligent and Connected Vehicles (Beijing) Research Institute Co., Ltd., which is also the National Innovation Center of Intelligent and Connected Vehicles. He received his B.S. degree majored in Automotive Engineering from Tsinghua University, China, in 2008, and his M.S. degree majored in Automotive Engineering from RWTH-Aachen, German and Ph.D. degree majored in Mechanical Engineering from Tsinghua University, China, in 2014.

Qiqige Wuniri, born in 1979, is currently a senior engineer at China Intelligent and Connected Vehicles (Beijing) Research Institute Co., Ltd. She received her Ph.D degree majored in Computer Science from Beihang University, China, in 2019. Xiaoping Du, born in 1962, is currently a professor with the School of Software at Beihang University, China, and Technical Director at China Intelligent and Connected Vehicles (Beijing) Research Institute Co., Ltd. He received his M.S. degree majored in Computer Science from from Chongqing University, China, in 1995, and his Ph.D. degree majored in Intelligent System from Kyushu University, Japan, in 2001

Qiuchi Xiong, born in 1991, is currently a senior engineer at China Intelligent and Connected Vehicles (Beijing) Research Institute Co., Ltd. He received his master degree majored in Mechanical Engineering from Virginia Polytechnic Institute and State University, USA, in 2020.

Tai Huang, born in 1990, is currently a senior engineer \& 6-sigma Black Belt engineer at China Intelligent and Connected Vehicles (Beijing) Research Institute Co., Ltd. He received his master degree majored in Communication Engineering from Chalmers University of Technology, Sweden, in 2016.

Keqiang Li, born in 1963, is currently an academician of Chinese Academy of Engineering, a Professor with the School of Vehicle and Mobility, Tsinghua University. He received his B.S. degrees majored in Mechanical Engineering from Tsinghua University, China, in 1985, and his M.S. degree and Ph.D. degree majored in Mechanical Engineering from Chongaing University, China, in 1988 and 1995, respectively. He has served as fellow member of Society of Automotive Engineers of China, editorial boards of International Journal of Vehicle Autonomous Systems, Chairperson of Expert Committee of CAICV (China Industrial Technology Innovation Strategic Alliance for ICVs), and CTO of CICV (China Intelligent and Connected Vehicles (Beijing) Research Institute Co., Ltd.).

\section{Funding}

Supported by Beijing Nova Program of Science and Technology (Grant No. Z191100001119087) and Beijing Municipal Science \& Technology Commission (Grant No. Z181100004618005 and Grant No. Z18111000460000)

\section{Competing Interests}

The authors declare no competing financial interests.

\section{Author Details}

${ }^{1}$ National Innovation Center of Intelligent and Connected Vehicles, Beijing 100176, China. ${ }^{2}$ School of Software, Beihang University, Beijing 100083 , China. ${ }^{3}$ State Key Laboratory of Automotive Safety \& Energy, School of Vehicle and Mobility, Tsinghua University, Beijing 100084, China. 
Received: 19 October 2020 Revised: 22 October 2021 Accepted: 7

November 2021

Published online: 16 December 2021

\section{References}

[1] A Talebpour, H S Mahmassani, Influence of connected and autonomous vehicles on traffic flow stability and throughput. Transportation Research Part C: Emerging Technologies, 2016, 71: 143-163.

[2] J J Q Yu, A Lam. Autonomous vehicle logistic system: Joint routing and charging strategy. IEEE Transactions on Intelligent Transportation Systems, 2017: 1-13.

[3] J Jeongmin, J Byungjin, KJ Choon et al. Autonomous robotic street sweeping: Initial attempt for curbside sweeping. IEEE International Conference on Consumer Electronics, Las Vegas, USA, 8-10 Jan. 2017: 72-73.

[4] C I Liu, P A loannou. A comparison of different AGV dispatching rules in an automated container terminal. International Conference on Intelligent Transportation Systems, Singapore, 3-6 Sept. 2002: 880-885.

[5] V Androulakis, J Sottile, S Schafrik et al. Concepts for development of autonomous coal mine shuttle cars. IEEE Transactions on Industry Applications, 2020, 56(3): 3272-3280.

[6] Y C Chou, H HC Chuang, B B M Shao. The impact of e-retail characteristics on initiating mobile retail services: A modular innovation perspective. Information \& Management, 2016, 53(4): 481-492.

[7] K M Gurumurthy, K M Kockelman, M D Simoni. Benefits and costs of ride-sharing in shared automated vehicles across Austin, Texas: opportunities for congestion pricing. Transportation Research Record, 2019, 2673(6): 548-556.

[8] K Stark, K Gade, D Heinrichs. What does the future of automated driving mean for public transportation. Transportation Research Record, 2019, 2673(2): 85-93.

[9] B Visnic. 2020 Hype cycle for connected and smart mobility. 1st Oct, 2020; https://www.sae.org/news/2020/09/2020-hype-cycle-forconnected-vehicles-and-smart-mobility

[10] H Farah, S Erkens, T P Alkim, et al. Infrastructure for automated and connected driving: State of the art and future research directions. Road Vehicle Automation, 2018, 4: 187-197.

[11] B Ran, P J Jin, D Boyce, et al. Perspectives on future transportation research: impact of intelligent transportation system technologies on next-generation transportation modeling. Journal of Intelligent Transportation Systems, 2012, 16(4): 226-242.

[12] K Li, Y Dai, S Li, et al. State-of-the-art and technical trends of intelligent and connected vehicles. Journal of Automotive Safety and Energy, 2017 8(1): 1-14.

[13] K Li, J Li, X Chang, et al. Principles and typical applications of cloud control system for intelligent and connected vehicles. Journal of Automotive Safety and Energy, 2020, 11(3): 261-275.

[14] S Kuzumaki. SIP-adus: Project reports. 2019.

[15] Korea Government, Developmental strategy for future vehicle industry: 2030 National Roadmap, 2019.

[16] ERTRAC. Connected automated driving roadmap. 2019.

[17] British Government. UK connected and automated mobility roadmap to $2030,2020$.

[18] Intelligent Transportation System Joint Program Office, Intelligent transportation systems strategic plan 2020-2025, 2020,

[19] National Development and Reform Commission of PRC, Innovative development strategy of intelligent vehicle, 2020.

[20] K Li, T Chen, Y Luo, et al. Intelligent environment-friendly vehicles: concept and case studies. IEEE Transactions on Intelligent Transportation Systems, 2012, 13(1): 318-328.

[21] G. Chinese. The strategy for innovation and development of intelligent vehicles. 1st Oct, 2020; https://www.ndrc.gov.cn/xxgk/zcfb/tz/202002/ t20200224_1221077.html.

[22] United States Department of Transportation,Office of the assistant secretary for research and technology (OST-R), 2020. [Online] Available: https://www.its.dot.gov/stratplan2020/index.htm.

[23] European Commission,Directorate-General for mobility and transport, 2019. [Online] Available: https://ec.europa.eu/transport/themes/its/cits_en.
[24] B Chang, J Chiou. Cloud computing-based analyses to predict vehicle driving shockwave for active safe driving in intelligent transportation system. IEEE Transactions on Intelligent Transportation Systems, 2020 21(2): 852-866.

[25] U Montanaro, S Fallah, M Dianati, et al. Cloud-assisted distributed control system architecture for platooning. 21st International Conference on Intelligent Transportation Systems, Maui, USA, 4-7 Nov. 2018: 1258-1265.

[26] K Katsaros, A Stevens, M Dianati, et al. Cooperative automation through the cloud: The CARMA project. ITS European Congress, Strasbourg, France, 19-22 June. 2017: 1-6.

[27] R Hussain, Z Rezaeifar, H Oh. A Paradigm shift from vehicular Ad Hoc networks to VANET-based clouds. Wireless Personal Communications, 2015, 83(2): 1131-1158.

[28] UK Department for Transport and the Department for Business, Energy \& Industrial Strategy,Centre for connected and autonomous vehicles, 2017. [Online] Available: https://www.connectedautomateddriving.eu/ wp-content/uploads/2017/10/2017_United-Kingdom_Connectedand-Automated-Vehicle-Research-and-Development-Projects.pdf.

[29] Volvo Car Group, Corporate communication,2019. [Online] Available: https://www.media.volvocars.com/global/en-gb/media/pressrelea ses/253968/volvo-cars-joins-groundbreaking-pan-european-safet y-data-sharing-pilot-project.

[30] Project Consortium Ko-HAF, Kooperatives hochautomatisiertes Fahren, 2019. [Online] Available: https://www.ko-haf.de/das-projekt/.

[31] S Olariu, M Eltoweissy, M Younis, Towards Autonomous Vehicular Clouds. ICST Transactions on Mobile Communications Applications, 2011, 11: e2.

[32] J Watson, M Pellerito, C Gladden, et al. Simulation and analysis of extended brake lights for inter-vehicle communication networks. 27th International Conference on Distributed Computing Systems Workshops, Toronto, Canada, 22-29 Jun. 2007: 87-87.

[33] P Barth, S Brummer, S Kienast, et al. Ko-HAF cooperative highly automated driving-contents of the project and focus of research. AmE-2017 - Automotive meets electronics, 2017: 1-10.

[34] CAICV. White paper on the system of coordinated control by vehicleroad-cloud integration. China Industry Innovation Alliance for the Intelligent and Connected Vehicles, 2020, 1(1): 1-33.

[35] HWang, H Zhao, J Zhang, et al. Survey on unmanned aerial vehicle networks: a cyber physical system perspective. IEEE Communications Surveys and Tutorials, 2020, 22(2): 1027-1070.

[36] P LI. Kyland technical paper for loT solutions. vol. 1, Kyland Technology Co., Ltd., https://www.kyland.com/materials/?page=2, 2020.

[37] P Sitbon, N Bulusu, W Feng. Urban-scale sensing for science. National Workshop for Research on High-Confidence Transportation Cyber-Physical Systems: Automotive, Aviation \& Rail, Washington DC, USA, 18-20 Nov. 2008.

[38] G Xiong, F Zhu, X Liu, et al. Cyber-physical-social system in intelligent transportation. IEEE/CAA Journal of Automatica Sinica, 2015, 2: 320-333.

[39] U Sanwal, O Hasan. Formal verification of cyber-physical systems: Coping with continuous elements. Proceedings of the 13th International Conference on Computer Science and its Applications, Ho Chi Minh City, Vietnam, 24-27 Jun. 2016: 358-371.

[40] A Gawanmeh, A Alwadi, S Parvin. Formal verification of control strategies for a cyber physical system. IEEE 37th International on Distributed Computering Systems Workshops, Atlanta, USA, 5-8 June 2017: 91-96.

[41] Nurjahan, F Nizam, S Chaki et al. Attack detection and prevention in the cyber physical system. International Conference on Computer Communication and Informatics, Coimbatore, India, 7-9 Jan. 2016: 1-6.

[42] K Evers, R Oram, S El-Tawab, et al. Security measurement on a cloudbased cyber-physical system used for Intelligent Transportation. 2017 IEEE International Conference on Vehicular Electronics and Safety (ICVES), Vienna, Austria, 27-28 Jun. 2017: 97-102.

[43] S Andrei, A Cheng. Optimizing automotive cyber-physical system specifications with multi-event dependencies. SYNASC 2008, 10th International Symposium on Symbolic and Numeric Algorithms for Scientific Computing, Timisoara, Romania, 26-29 Sept. 2008: 475-479.

[44] A Platzer. Verification of cyberphysical transportation systems. IEEE Inte/ligent Systems, 2009, 24(4): 10-13.

[45] Y Wang, X Yang, $\mathrm{H}$ Liang, et al. A review of the self-adaptive traffic signal control system based on future traffic environment. Journal of Advanced Transportation, 2018: 1-12. 
[46] P Garraghan, D McKee, X Ouyang, et al. SEED: A scalable approach for cyber-physical system simulation. IEEE Transactions on Services Computing, 2016, 9(2): 199-212.

[47] J Wan, A Canedo, M A A Faruque. Cyber-physical codesign at the functional level for multidomain automotive systems. IEEE Systems Journal, 2017, 11(4): 2949-2959.

[48] D Shin, S He, J Zhang. Robust and cost-effective design of cyber-physical systems: an optimal middleware deployment approach. IEEE/ACM Transactions on Networking, 2016, 24(2): 1081-1094.

[49] CAICV, CPS reference architecture for ICV, China Industry Innovation Alliance for the Intelligent and Connected Vehicles, 2020, 1.

[50] A M Madni, M Sievers. Model-based systems engineering: motivation, current status, and research opportunities. Systems Engineering, 2018, 21(3): 172-190.

[51] A C Pang, E Au, B Ai, et al. Guest editorial introduction to the special section on fog/edge computing for autonomous and connected cars. IEEE Transactions Vehicular Technology, 2019, 68(4): 3059-3060.

[52] CYou, K Huang, $\mathrm{H}$ Chae, et al. Energy-efficient resource allocation for mobile-edge computation offloading. IEEE Transactions on Wireless Communications, 2017, 16(3): 1397-1411.

[53] CYou, K Huang, H Chae. Energy efficient mobile cloud computing powered by wireless energy transfer. IEEE Journal on Selected Areas in Communications, 2016, 34(5): 1757-1771.

[54] K Kumar, J Liu, Y H Lu, et al. A survey of computation offloading for mobile systems. Mobile Networks and Applications, 2013, 18:129-140.

[55] SE Mahmoodi, R N Uma, K P Subbalakshmi. Optimal joint scheduling and cloud offloading for mobile applications. IEEE Transactions on Cloud Computing, 2019, 7(2): 301-313,

[56] J Kwak, Y Kim, J Lee, et al. DREAM: dynamic resource and task allocation for energy minimization in mobile cloud systems. IEEE Journal on Selected Areas in Communications, 2015, 33(12): 2510-2523.

[57] KWang, K Yang, C S Magurawalage. Joint energy minimization and resource allocation in C-RAN with mobile cloud. IEEE Transactions on Cloud Computing, 2018, 6(3): 760-770.

[58] L Lei, Z Zhong, K Zheng, et al. Challenges on wireless heterogeneous networks for mobile cloud computing. IEEE Wireless Communications, 2013, 20(3): 34-44

[59] R Kaewpuang, D Niyato, P Wang, et al. A framework for cooperative resource management in mobile cloud computing. IEEE Journal on Selected Areas in Communications, 2013, 31(12): 2685-2700.

[60] Y Ge, Y Zhang, Q Qiu, et al. A game theoretic resource allocation for overall energy minimization in mobile cloud computing system. Proceedings of the International Symposium on Low Power Electronics and Design, Redondo, USA, 30 Jul.- Aug. 2012: 279-284,

[61] M S Elbamby, M Bennis, W Saad. Proactive edge computing in latencyconstrained fog networks. 2017 European Conference on Networks and Communications, Oulu, Finand, 12-15 Jun. 2017: 1-6.

[62] A Mukhtar, L Xia, T B Tang. Vehicle detection techniques for collision avoidance systems: a review. IEEE Transactions on Intelligent Transportation Systems. 2015, 16(5): 1-21.

[63] C Chen, A Seff, A Kornhauser, et al. DeepDriving: Learning affordance for direct perception in autonomous driving. International Conference on Computer Vision, Santiago, Chile, 11-18 Dec. 2015: 2722-2730.

[64] Y LeCun, Y Bengio, G Hinton. Deep learning. Nature, 2015, 521 (7553): $436-444$

[65] $X . H u, X X u, Y X i a o$, et al. SINet: a scale-insensitive convolutional neural network for fast vehicle detection. IEEE Transactions on Intelligent Transportation Systems, 2019, 20(3): 1010-1019.

[66] Y He, HWang, B Zhang, Color-based road detection in urban traffic scenes. IEEE Transactions on Intelligent Transportation Systems, 2004, 5(4): 309-318.

[67] HY Cheng, B S Jeng, PT Tseng, et al. Lane detection with moving vehicles in the traffic scenes. IEEE Transactions on Intelligent Transportation Systems, 2006, 7(4): 571-582.

[68] Z Zhu, D Liang, S Zhang, et al. Traffic-sign detection and classification in the wild. IEEE Conference on Computer Vision and Pattern Recognition, Las Vegas, USA, 27-30 Jun. 2016: 2110-2118.

[69] A Geiger, P Lenz, C Stiller, et al. Vision meets robotics: the KITTI dataset. The International Journal of Robotics Research, 2013, 32(11): 1231-1237.
[70] X Huang, X Cheng, Q Geng, et al. The ApolloScape dataset for autonomous driving. IEEE/CVF Conference on Computer Vision and Pattern Recognition Workshops, Salt Lake City, USA, 18-22 Jun. 2018: 954-960.

[71] Y Lou, Y Bai, J Liu, et al. VERI-Wild: A large dataset and a new method for vehicle re-identification in the wild. IEEE/CVF Conference on Computer Vision and Pattern Recognition, Long Beach, USA, 15-20 Jun. 2019: 3230-3238.

[72] A Krizhevsky, I Sutskever, G Hinton. ImageNet classification with deep convolutional neural networks. Communications of the ACM, 2017, 60(6): 84-90.

[73] A Bochkovskiy, CY Wang, HY M Liao. YOLOv4: optimal speed and accuracy of object detection. 2020. arXiv:2004.10934.

[74] O Vinyals, A Toshev, S Bengio, et al. Show and tell: lessons learned from the 2015 MSCOCO image captioning challenge. IEEE Transactions on Pattern Analysis and Machine Intelligence, 2017, 39(4): 652-663.

[75] XWu, D Sahoo, SC H Hoi. Recent advances in deep learning for object detection. Neurocomputing, 2020, 396: 39-64.

[76] G Ciaparrone, F Luque Sánchez, S Tabik, et al. Deep learning in video multi-object tracking: A survey. Neurocomputing, 2019: 381.

[77] S Sivaraman, M M Trivedi. Looking at vehicles on the road: a survey of vision-based vehicle detection, tracking, and behavior analysis. IEEE Transactions on Intelligent Transportation Systems, 2013, 14(4): 1773-1795.

[78] J H Lemelson, R D Pedersen. GPS vehicle collision avoidance warning and control system and method. 2001.

[79] B Khaleghi, A Khamis, F O Karray, et al. Multisensor data fusion: a review of the state-of-the-art. Information Fusion, 2013, 14(1): 28-44.

[80] W Zhang. LIDAR-based road and road-edge detection. IEEE Intelligent Vehicles Symposium, La Jolla, USA, 21-24 Jun. 2010: 845-848.

[81] K Kidono, T Miyasaka, A Watanabe, et al. Pedestrian recognition using high-definition LIDAR. IEEE Intelligent Vehicles Symposium, Baden-Baden, Germany, 5-9 Jun. 2011: 405-410.

[82] K Takagi, K Morikawa, T Ogawa, et al. Road environment recognition using on-vehicle LIDAR. IEEE Intelligent Vehicles Symposium, Meguro-Ku, Japan, 13-15 Jun. 2006: 120-125.

[83] J Wu, H Xu, J Zheng. Automatic background filtering and lane identification with roadside LiDAR data. IEEE 20th International Conference on Intelligent Transportation Systems, Yokohama, Japan, 16-19 Oct. 2017: $1-6$.

[84] J Zhao, H Xu, H Liu, et al. Detection and tracking of pedestrians and vehicles using roadside LiDAR sensors. Transportation Research Part C Emerging Technologies, 2019, 100: 68-87.

[85] J Choi, S Ulbrich, B Lichte, et al. Multi-target tracking using a 3D-Lidar sensor for autonomous vehicles. 16th International IEEE Conference on Intelligent Transportation Systems, The Hague, Netherlands, 6-9 Oct. 2013: 881-886.

[86] J Zhao, H Xu, JWu, et al. Trajectory tracking and prediction of pedestrian's crossing intention using roadside LiDAR. IET Intelligent Transport Systems, 2019, 13(5): 789-795.

[87] J Hasch, E Topak, R Schnabel, et al. Millimeter-Wave technology for automotive radar sensors in the $77 \mathrm{GHz}$ frequency band. IEEE Transactions on Microwave Theory and Techniques, 2012, 60(3): 845-860.

[88] STokoro, K Kuroda, A Kawakubo, et al. Electronically scanned millimeter-wave radar for pre-crash safety and adaptive cruise control system. Proceedings of IEEE IV2003 Intelligent Vehicles Symposium, Columbus, USA, 9-11 Jun. 2003: 304-309.

[89] SR E Datondji, Y Dupuis, P Subirats, et al. A survey of vision-based traffic monitoring of road intersections. IEEE Transactions on Intelligent Transportation Systems, 2016, 17(10): 2681-2698.

[90] P Wei, L Cagle, T Reza, et al. LiDAR and camera detection fusion in a real-time industrial multi-sensor collision avoidance system. Electronics, 2018, 7(6): 84

[91] F Zhang, D Clarke, A C Knoll. Vehicle detection based on LiDAR and camera fusion. 17th International IEEE Conference on Intelligent Transportation Systems, Qingdao, China, 8-11 Oct. 2014: 1620-1625.

[92] M Meyer, G Kuschk. Deep learning based 3D object detection for automotive radar and camera. European Radar Conference, Paris, France, 2-4 Oct. 2019: 133-136. 
[93] Z Sun, G Bebis, R Miller. On-road vehicle detection using optical sensors: a review. Proceedings of 7 th International IEEE Conference on Intelligent Transportation Systems, Washington, USA, 3-6 Oct. 2004: 585-590.

[94] M Enzweiler, D M Gavrila. Monocular pedestrian detection: survey and experiments. IEEE Transactions on Pattern Analysis and Machine Intelligence, 2009, 31(12): 2179-2195.

[95] STaghvaeeyan, R Rajamani. Portable roadside sensors for vehicle counting, classification, and speed measurement. IEEE Transactions on Intelligent Transportation Systems, 2014, 15(1): 73-83.

[96] L Yang, M Li, X Song, et al. Vehicle speed measurement based on binocular stereovision system. IEEE Access, 2019, 7: 106628-106641.

[97] M Samadi, M F Othman. Using fish-eye stereo vision system for autonomous vehicles. 11th Asia Control Conference, Gold Coast, Australia, 17-20 Dec. 2017: 1331-1334.

[98] L Chen, Q Zou, Z Pan, et al. Surrounding vehicle detection using an FPGA panoramic camera and deep CNNs. IEEE Transactions on Intelligent Transportation Systems, 2019: 1-13.

[99] R Gade, T B Moeslund. Thermal cameras and applications: a survey. Machine Vision Applications, 2014, 25: 245-262.

[100] K Rumar. Infrared night vision systems and driver needs. SAE 2003 World Congress \& Exhibition, Warrendale, USA, 3 Mar. 2003.

[101] M Klotz, H Rohling. $24 \mathrm{GHz}$ radar sensors for automotive applications. Proceedings of 13 th International Conference on Microwaves, Radar \& Wireless Communications, Wroclaw, Poland, 22-24 May 2000: 359-362.

[102] H Rohling, M M Meinecke. Waveform design principles for automotive radar systems. 2001 CIE International Conference on Radar, Beijing, China, 15-18 Oct. 2001: 1-4.

[103] D Gruver, PY Droz, G Pennecot, et al. Vehicle with multiple light detection and ranging devices (LIDARs), 2017.

[104] D S Hall. High definition lidar system, 2007.

[105] SThrun, M Montemerlo, H Dahlkamp et al. Stanley: the robot that won the DARPA grand challenge. Journal of Field Robotics, 2006, 23(9): 661-692.

[106] HW Yoo, N Druml, D Brunner, et al. MEMS-based Lidar for autonomous driving. Elektrotechnik and Informationstechnik, 2018, 135(6): 408-415.

[107] T O Chan, D D Lichti, D Belton. Temporal analysis and automatic calibration of the velodyne HDL-32E lidar system. Isprs Annals of the Photogrammetry Remote Sensing and Spatial Information Sciences, 2013: 61-66.

[108] C L Glennie, D D Lichti. Static calibration and analysis of the Velodyne HDL-64E S2 for high accuracy mobile scanning. Remote Sensing, 2010, 2(6): 1610-1624

[109] N Jiang, Y Xu, Z Zhou, et al. Multi-attribute driven vehicle re-identification with spatial-temporal re-ranking. 25th IEEE International Conference on Image Processing, Athens, Greece, 7-10 Oct. 2018: 858-862.

[110] Z Wang, L Tang, X Liu, et al. Orientation invariant feature embedding and spatial temporal regularization for vehicle re-identification. IEEE International Conference on Computer Vision, Venice, Italy, 22-27 Oct. 2017: 379-387.

[111] J Tang, Y Zhu, Y Huang, et al. Identification and interpretation of spatialtemporal mismatch between taxi demand and supply using global positioning system data. Journal of Intelligent Transportation Systems, 2019, 23(4): 403-415.

[112] D F Cordry. Automatic speed control for automotive vehicles. United States, to Cordry, Durrel F., 1956.

[113] M Liubakka, D Rhode, J Winkelman. Adaptive automotive speed control. Proceedings of the American Control Conference, Boston, Massachusetts, June 2006: 1-26.

[114] K Oda, H Takeuchi, M Tsujii, et al. Practical estimator for self-tuning automotive cruise control. American Control Conference, 26-28 June 1991: 2066-2071

[115] M Tsujii, H Takeuchi, K Oda, et al. Application of self-tuning to automotive cruise control. American Control Conference, Boston, USA, 26-28 Jun. 1990: 1843-1848.

[116] S Björnander, L Grunske. C4-01 TR M50, Adaptive cruise controllers - a literature review. Faculty of Information and Communications Technologies, Swinburne University of Technology, Australia, 2008.

[117] E Montroll. Traffic dynamics: studies in car following. Operations Research, 1958, 6: 165-184.

[118] K Junaid, W Shuning, K Usman, et al. LQR autonomous longitudinal cruise control with a minimum order state observer. Proceedings of the
Eighth IASTED International Conference on Intelligent Systems and Control, Cambridge, USA, 31 Oct.—2 Nov. 2006.

[119] V L Bageshwar, W L Garrard, R Rajamani. Model predictive control of transitional maneuvers for adaptive cruise control vehicles. IEEE Transactions on Vehicular Technology, 2004, 53(5): 1573-1585.

[120] D Corona, M Lazar, B D Schutter, et al. A hybrid MPC approach to the design of a smart adaptive cruise controller. IEEE International Conference on Control Applications, Munich, Germany, 4-6 Oct. 2006: 231-236.

[121] C Zhao, W Xiang, P Richardson. Vehicle lateral control and yaw stability control through differential braking. IEEE International Symposium on Industrial Electronics, Montreal, Canada, 9-13 July 2006: 384-389.

[122] Jihao Luo, P Tsiotras. Control design for systems in chained form with bounded inputs. Proceedings of the 1988 American Control Conference, Philadelphia, PA, USA, 26-26 June 1998: 473-477.

[123] H Guo, F Liu, F Xu, et al. Nonlinear model predictive lateral stability control of active chassis for intelligent vehicles and its FPGA implementation. IEEE Transactions on Systems, Man, and Cybernetics: Systems, 2019, 49(1): 2-13

[124] R Fan, J Jiao, H Ye, et al. Key ingredients of self-driving cars. EUSIPCO 2019 Satellite Workshop: Signal Processing, Computer Vision and Deep Learning for Autonomous Systems, A Coruna, Spain, 6 Sept. 2019.

[125] Q Yao, Y Tian, Q Wang, et al. Control strategies on path tracking for autonomous vehicle: state of the art and future challenges. IEEE Access, 2020, 8: 161211-161222.

[126] M W Park, S W Lee, WY Han. Development of lateral control system for autonomous vehicle based on adaptive pure pursuit algorithm. 14th International Conference on Control, Automation and Systems, Gyeonggido, Korea, 22-25 Oct. 2014: 1443-1447.

[127] N H Amer, K Hudha, H Zamzuri. Adaptive modified Stanley controller with fuzzy supervisory system for trajectory tracking of an autonomous armoured vehicle. Robotics and Autonomous Systems, 2018, 105: 94-111.

[128] N Ame, K Hudha, H Zamzuri. Adaptive trajectory tracking controller for an armoured vehicle: hard ware-in-the-loop simulation. 57th Annual Conference of the Society of Instrument and Control Engineers of Japan (SICE), Nara, Japan, 11-14 Sept. 2018: 462-467.

[129] G Han, W Fu, W Wang, et al. The lateral tracking control for the intelligent vehicle based on adaptive PID neural network. Sensors, 2017, 17(6): 1244.

[130] R Potluri, A K. Singh. Path-tracking control of an autonomous 4WS4WD electric vehicle using its natural feedback loops. IEEE Transactions on Control Systems Technology, 2015, 23(5): 2053-2062.

[131] A. Al-Mayyahi, W Wang, P Birch. Path tracking of autonomous ground vehicle based on fractional order PID controller optimized by PSO. 13th IEEE International Symposium on Applied Machine Intelligence \& Informatics, Herl'any, Slovakia, 22-24 Jan. 2015: 105-109.

[132] Y Zennir, S Allou. Comparison of PID and fuzzy controller for path tracking control of autonomous electrical vehicles. International Conference on Electrical Sciences and Technologies in Maghreb, Algiers, Algeria, 28-31 Oct. 2018: 1-6.

[133] TTian, Z Hou, S Liu, et al. Model-free adaptive control based lateral control of self-driving car. Acta Automatica Sinica, 2017, 43(11): 1931-1940.

[134] S Liu, Z Hou, T Tian, et al. A novel dual successive projection-based model-free adaptive control method and application to an autonomous car. IEEE transactions on neural networks and learning systems, 2019, 30(11): 3444-3457.

[135] N Li, I Kolmanovsky. Model-free optimal control based automotive control system falsification, American Control Conference (ACC), Seattle, USA, 24-26 May. 2017: 636-641.

[136] F Lin, L Ni, Y Zhao, et al. Path following control of intelligent vehicles considering lateral stability. Journal of South China University of Technology (Natural Science Edition), 2018, 46(1): 78-84.

[137] X Zhang, X Zhu. Autonomous path tracking control of intelligent electric vehicles based on lane detection and optimal preview method. Expert Systems with Applications, 2019, 121: 38-48.

[138] C Chatzikomis, A Sorniotti, P Gruber, Comparison of path tracking and torque-vectoring controllers for autonomous electric vehicles. IEEE Transactions on Intelligent Vehicles, 2018, 3(4): 559-571.

[139] $\mathrm{Y} \mathrm{Li}, J \mathrm{Ni}, \mathrm{J} \mathrm{Hu}$. The design of driverless vehicle trajectory tracking control strategy. IFAC-PapersOnLine, 2018, 51(31): 738-745. 
[140] N R Kapania, J C Gerdes. Design of a feedback-feedforward steering controller for accurate path tracking and stability at the limits of handling, Vehicle System Dynamics, 2015, 53(12): 1687-1704.

[141] Q Tan, P Dai, Z Zhang, et al., MPC and PSO based control methodology for path tracking of 4WS4WD vehicles. Applied Sciences, 2018, 8(6):1000.

[142] J. Ji, A Khajepour. Path planning and tracking for vehicle collision avoidance based on model predictive control with multiconstraints. IEEE Transactions on Vehicular Technology, 2017, 25(24): 86-97.

[143] C Shen, H Guo, F Liu, et al. MPC-based path tracking controller design for autonomous ground vehicles. 36th Chinese Control Conference (CCC), Dalian, China, 26-28 July 2017: 9584-9589.

[144] H Jing, C Hu, F Yan, et al. Robust $H \infty$ output-feedback control for path following of autonomous ground vehicles. 54th IEEE Conference on Decision \& Control (CDC), 2015: 1515-1520.

[145] S Hong, C Zhang, G An, Fuzzy-model-based Ho dynamic output feedback control with feedforward for autonomous vehicle path tracking. International Conference on Fuzzy Theory and Its Applications (iFUZZY), 2017: 1-6.

[146] C. Hu, H. Jing, R. Wang. Robust $\mathrm{H} \infty$ output-feedback control for path following of autonomous ground vehicles. Mechanical Systems and Signal Processing, 2016, 70: 414-427.

[147] D Ren, J Zhang, J Zhang. Sliding mode control for vehicle following with parametric uncertainty. Electric Machines and Control, 2010, 14(1): 73-78.

[148] RWang, G Yin, X Jin. Robust adaptive sliding mode control for nonlinear four-wheel steering autonomous vehicles path tracking systems. 8th Power Electronics \& Motion Control Conference, Hefei, China, 22-26 May 2016: 2999-3006.

[149] J Guo, Y Luo, K Li. Adaptive fuzzy sliding mode control for coordinated longitudinal and lateral motions of multiple autonomous vehicles in a platoon. Science China(Technological Sciences), 2017, 60(4): 576-586.

[150] T Sun, Y Pan, J Zhang, et al. Robust model predictive control for constrained continuous-time nonlinear systems. International Journal of Control, 2018, 91(2): 359-368.

[151] R Gonzalez, M Fiacchini, T Alamo, et al. Online robust tube-based MPC for time-varying systems: a practical approach. International Journal of Control, 2011, 84(6): 1157-1170.

[152] I Nodozi, M Rahmani. LMI-based robust mixed-integer model predictive control for hybrid systems. International Journal of Control, 2019, 12(20): 12-22.

[153] X Wang, Y Zhang, Z Xue. Fuzzy sliding mode control based on RBF neural network for AUV path tracking. International Conference on Intelligent Robotics and Applications, Switzerland: Springer, Cham, 2019: 637-648.

[154] Y Chen, Z Li, H Kong. Model predictive tracking control of nonholonomic mobile robots with coupled input constraints and unknown dynamics. IEEE Transactions on Industrial Informatics, 2019, 15(6): 3198-3205.

[155] N Wang, Z Sun, J Yin, et al. Fuzzy unknown observer-based robust adaptive path following control of underactuated surface vehicles subject to multiple unknowns. Ocean Engineering, 2019, 176: 57-64.

[156] M Ahmadian, S Southward. No-Jerk skyhook control methods for semiactive suspensions. Journal of Vibration and Acoustics, 2004, 126(4): 580-584

[157] Y Liu, L Zuo. Mixed skyhook and power-driven-damper: a new low-jerk semi-active suspension control based on power flow analysis. Journal of Dynamic Systems, Measurement, and Control, 2016, 138(8): 081009.

[158] Q Xiong, B Qin, X Li, et al. A rule-based damping control of MMR-based energy-harvesting vehicle suspension. 2020 American Control Conference (ACC), Denver, USA, 1-3 July 2020: 2262-2267.

[159] S Guo, Q Xiong, J M Li, et al. Truck suspension control for pavement protection at signalized intersections. 2018 Annual American Control Conference (ACC), Milwaukee, USA, 27-29 June 2018: 3696-3701.

[160] H Fritz. Longitudinal and lateral control of heavy duty trucks for automated vehicle following in mixed traffic: experimental results from the CHAUFFEUR project. Kohala Coast, USA, 22-27 Aug. 1999: 1348-1352.

[161] H Peng, M Tomizuka. Preview control for vehicle lateral guidance in highway automation. American Control Conference, Boston, USA, 26-28 Jun. 1991: 3090-3095.

[162] L Li, D Wen, D Yao. A survey of traffic control with vehicular communications. IEEE Transactions on Intelligent Transportation Systems, 2014, 15(1): 425-432.
[163] Q Guo, L Li, X Ban. Urban traffic signal control with connected and automated vehicles: a survey. Transportation Research Part C: Emerging Technologies, 2019, 101: 313-334.

[164] W Li, X Ban. Traffic signal timing optimization in connected vehicles environment. 2017 IEEE Intelligent Vehicles Symposium (IV), Los Angeles, CA, USA, 11-14 June 2017: 1330-1335.

[165] Y Zheng, J Wang, K Li. Smoothing traffic flow via control of autonomous vehicles. IEEE Internet of Things Journal, 2020, 7(5): 3882-3896.

[166] J Wang, Y Zheng, Q Xu, et al. Controllability analysis and optimal control of mixed traffic flow with human-driven and autonomous vehicles. IEEE Transactions on Intelligent Transportation Systems, 2020: 1-15.

[167] Y Bian, S E Li, W Ren, et al. Cooperation of multiple connected vehicles at unsignalized intersections: distributed observation, optimization, and control. IEEE Transactions on Industrial Electronics, 2020, 67(12): 10744-10754.

[168] M Cai, Q Xu, K Li, et al. Multi-lane formation assignment and control for connected vehicles. IEEE Intelligent Vehicles Symposium, Paris, France, 9-12 June 2019: 1968-1973.

[169] M Goldhammer, E Strigel, D Meissner, et al. Cooperative multi sensor network for traffic safety applications at intersections. International Conference on Intelligent Transportation Systems, Anchorage, USA, 16-19 Sept. 2012: 1178-1183.

[170] SW Kim, B Qin, Z J Chong, et al. Multivehicle cooperative driving using cooperative perception: design and experimental validation. IEEE Transactions on Intelligent Transportation Systems, 2015, 16(2): 663-680.

[171] Q Chen, S Tang, Q Yang, et al. Cooper: cooperative perception for connected autonomous vehicles based on 3D point clouds. 39th IEEE International Conference on Distributed Computing Systems, Dallas, USA, 7-10 July 2019: 514-524.

[172] M Gerla, E Lee, G Pau, et al. Internet of vehicles: from intelligent grid to autonomous cars and vehicular clouds. International Journal of Distributed Sensor Networs, 2016, 12(9): 241-246.

[173] B N Krishna. Study of ad hoc networks with reference to MANET, VANET, FANET. International Journal of Advanced Research in Computer Science and Software Engineering, 2017, 7(7): 390-394.

[174] V Kumar, N Chand, S Mishra. Applications of VANETs: present \& future. Communications and Network, 2013, 5: 12-15.

[175] P Papadimitratos, A D L Fortelle, K Evenssen, et al. Vehicular communication systems: enabling technologies, applications, and future outlook on intelligent transportation. IEEE Communications Magazine, 2009, 47(11): 84-95

[176] S Al-Sultan, M M Al-Doori, A H Al-Bayatti, et al. A comprehensive survey on vehicular ad hoc network. Journal of Network and Computer Applications, 2014, 37: 380-392.

[177] M J Booysen, S Zeadally, G J v Rooyen. Survey of media access control protocols for vehicular ad hoc networks. IET Communications, 2011, 5(11): 1619-1631.

[178] T L Willke, P Tientrakool, N F Maxemchuk. A survey of inter-vehicle communication protocols and their applications. IEEE Communications Surveys \& Tutorials, 2009, 11(2): 3-20.

[179] G Araniti, C Campolo, M Condoluci, et al. LTE for vehicular networking: a survey. IEEE Communications Magazine, 2013, 51(5): 148-157.

[180] J Liu, N Kato, J Ma, et al. Device-to-Device communication in LTEAdvanced networks: a survey. IEEE Communications Surveys \& Tutorials, 2015, 17(4): 1923-1940.

[181] L Liu, W Yu. A D2D-based protocol for ultra-reliable wireless communications for industrial automation. IEEE Transactions on Wireless Communications, 2018, 17(8): 5045-5058.

[182] J B Kenney. Dedicated short-range communications (DSRC) standards in the United States. Proceedings of the IEEE, 2011, 99(7): 1162-1182.

[183] S Chen, J Hu, Y Shi, et al. LTE-V: a TD-LTE-based V2X solution for future vehicular network. IEEE Internet of Things Journal, 2016, 3(6): 997-1005.

[184] S Sun, J Hu, Y Peng, et al. Support for vehicle-to-everything services based on LTE. IEEE Wireless Communications, 2016, 23(3): 4-8.

[185] R Aslani, E Saberinia, M. Rasti. Resource allocation for cellular V2X networks mode-3 with underlay approach in LTE-V standard. IEEE Transactions on Vehicular Technology, 2020, 69(8): 8601-8612.

[186] AutoTalks. DSRC vs. C-V2X for safety applications, Oct. 2020. [Online] Available: https://auto-talks.com/dsrc-vs-c-v2x/.

[187] Qualcomm. Leading the world to 5G: Cellular Vehicle-to Everything (C-V2X) technologies, June 2016. [Online] Available: https://www.qualc 
omm.com/media/documents/files/cellular-vehicle-to-everything -c-v2x-technologies.pdf.

[188] D Yang, Q Li, W Peng. Discussion Issues in the development of automatic driving map and positioning industry. 8th Annual Conference on China Satellite Navigation \& Positioning Services and China Beidou Applications, Zhengzhou, China, 10 Sept. 2019: 14-22.

[189] J Liu, J Xiao, H Cao, et al. The status and challenges of high precision map for automated driving. Proceedings of China Satellite Navigation Conference, Beijing, China, 22-25 May 2019: 266-276.

[190] M Dupuis, M Strobl, H Grezlikowski. OpenDRIVE 2010 and beyond status and future of the de facto standard for the description of road networks, Proceedings of the Driving Simulation Conference Europe, Paris, France, 9-10 Sept. 2010: 231-242.

[191] C Katrakazas, M A Quddus, W H Chen, et al. Real-time motion planning methods for autonomous on-road driving: state-of-the-art and future research directions. Transportation Research Part C Emerging Technologies, 2015, 60: 416-442.

[192] S Kuutti, S Fallah, K Katsaros, et al. A survey of the state-of-the-art localization techniques and their potentials for autonomous vehicle applications. IEEE Internet of Things Journal, 2018, 5(2): 829-846.

[193] T Menzel, G Bagschik, L Isensee, et al. From functional to logical scenarios: detailing a keyword-based scenario description for execution in a simulation environment. IEEE Intelligent Vehicles Symposium, Paris, France 9-12 June 2019: 2383-2390.

[194] J Jullien, C Martel, L Vignollet, et al. OpenScenario: a flexible integrated environment to develop educational activities based on pedagogical scenarios. 9th IEEE InternationalConference on Advanced Learning Technologies, Riga, Latvia, 15-17 July 2009: 509-513.

[195] Y Laschinsky, K v Neumann-Cosel, M Gonter, et al. Evaluation of an active safety light using virtual test drive within vehicle in the loop. IEEE International Conference on Industrial Terchnologies, Viña del Mar, Chile, 14-17 Mar. 2010: 1119-1112.

\section{Submit your manuscript to a SpringerOpen ${ }^{\circ}$ journal and benefit from:}

- Convenient online submission

- Rigorous peer review

- Open access: articles freely available online

- High visibility within the field

- Retaining the copyright to your article

Submit your next manuscript at $\gg$ springeropen.com 